\title{
On the geometry of the complex quadric
}

Dedicated to Professor Noboru Tanaka on his 60th birthday

\author{
Jacques GASQUI and Hubert GOLDSCHMIDT ${ }^{1}$ \\ (Received May 21, 1990)
}

\section{Introduction.}

In this paper, we present various results concerning the geometry of the complex quadric $Q_{n}$ of dimension $n \geq 3$ which are needed in the study of the infinitesimal rigidity of this space. We consider $Q_{n}$ both as a complex hypersurface of the complex projective space $\boldsymbol{C} \boldsymbol{P}^{n+1}$ and as a symmetric space.

Following [15], we introduce the real structure $K_{\nu}$ of the quadric $Q_{n}$, corresponding to a unit normal vector $\nu$ of this hypersurface of $\boldsymbol{C P}^{n+1}$ at $x \in Q_{n}$, which is an involution of the tangent space $T_{x}$ of $Q_{n}$ at $x$. This involution $K_{\nu}$ determines a decomposition

$$
T_{x}=T_{\nu, x}^{+} \oplus T_{\nu, x}^{-}
$$

into two $n$-dimensional components. We say that the real structures of $Q_{n}$ are oriented if, for all unit normals $\nu$ to $Q_{n}$ at $x \in Q_{n}$, the subspaces $T_{\nu, x}^{+}$admit orientations which are compatible with the action of the group $G=S O(n+2)$ of isometries of $Q_{n}$. We show that the real structures of $Q_{n}$ are orientable if and only if $n$ is even. If $n=4$, to such an orientation of these real structures corresponds a $*$-operator, which is an involution of a sub-bundle of the bundle of symmetric 2 -forms on $Q_{4}$; it is analogous to the usual involution of exterior 2 -forms on oriented Riemannian manifolds of dimension 4 . In $\S 3$, we use these real structures and this $*$-operator to decompose the bundle $S^{2} T_{c}^{*}$ of complex-valued symmetric 2-forms on $Q_{n}$ into irreducible $G$-invariant sub-bundles.

A symmetric 2 -form on a compact symmetric space $(X, g)$ satisfies the zero-energy condition if all its integrals along the closed geodesics of $X$ vanish. The space $(X, g)$ is infinitesimally rigid if the only symmetric 2 -forms on $X$ satisfying the zero-energy condition are the Lie derivatives of the metric $g$.

In order to study the infinitesimal rigidity of $Q_{n}$, with $n \geq 4$, we first

${ }_{1}$ The second author was supported in part by NSF Grant DMS 87-04209. 
consider the family $\mathscr{F}$ of totally geodesic surfaces of $Q_{n}$ contained in totally geodesic submanifolds of $Q_{n}$, which are already known to be infinitesimally rigid. If $n \geq 4$, according to Dieng [2], $\mathscr{F}$ consists of the totally geodesic submanifolds of $Q_{n}$, isometric either to a complex projective line of maximal curvature, to a flat torus of dimension 2 or to a real projective plane. These complex projective lines and these real projective planes are contained in totally geodesic complex projective planes. The infinitesimal rigidity of flat tori and complex projective planes was proved by Michel ([13], [14]) and Tsukamoto [17] (see also [5] and [6]). To exploit the fact that these submanifolds of $Q_{n}$ are infinitesimally rigid, we introduce the bundle $N$ of curvature-like tensors of type $(0,4)$ which vanish when restricted to the surfaces of the family $\mathscr{F}$. The infinitesimal orbit of the curvature $\widetilde{G}$ of $Q_{n}$, defined in [4] for any symmetric space, is a sub-bundle of $N$. We require a description of $N$ and, in particular, an explicit complement of $\widetilde{G}$ in $N$. This is possible if one is able to find a suitable bound for the rank of the bundle $N$. One of the goals of this paper is to present our computations announced and used in [6] leading to such a bound, when $n \geq 5$ (Proposition 5.2). This is a crucial step in our proof of the infinitesimal rigidity of $Q_{n}$, with $n \geq 5$, given there; it permits us to avoid the use of the representation theory of the group $G$ and harmonic analysis on its homogeneous space $Q_{n}$.

When $n=4$, this bound fails to hold and a complement of $\widetilde{G}$ in $N$ has additional components in this case. The methods used in [6] break down and a new approach is necessary.

We propose a new method, inspired in part by Michel's work [13], combining certain aspects of the techniques of [6] with those of [5], which we shall use in a future publication to analyze the infinitesimal rigidity problem for $Q_{4}$ and which we now briefly describe; we also point out the relevant results which are proved here. The bundle $N$ is still of paramount importance. Rather than a complete analysis of $N$, in this case we only need a characterization of the bundle $\operatorname{Tr} N$ of symmetric 2 -forms which are traces of elements of $N$. Here, we determine the bundle $\operatorname{Tr} N$ for $Q_{4}$ in terms of the decomposition of $S^{2} T_{C}^{*}$ into irreducible factors, and show that its elements are traceless symmetric 2-forms (Proposition 5.1). This computation is substantially simpler than the one giving us the rank of $N$ when $n \geq 5$.

On an irreducible symmetric space $X$, the resolution of the sheaf of Killing vector field introduced in [4] is related to the complex of [3] on the Einstein manifold $X$, in which the linearization of the Ricci operator appears. We recall that this operator involves the Lichnerowicz La- 
placian $\Delta$ acting on symmetric 2 -forms, These facts, taken together with the descriptions of $\operatorname{Tr} N$ and of the action of the operator $\Delta$ on the space $C^{\infty}\left(S^{2} T_{C}^{*}\right)$ of complex-valued symmetric 2 -forms on $Q_{4}$, play an essential role in this approach to the infinitesimal rigidity question for $Q_{4}$. We also require the representation theory of the group $G$ of isometries of our space, and the decomposition of $C^{\infty}\left(S^{2} T_{C}^{*}\right)$ into irreducible $G$-modules, just as we did in [5] for the complex projective spaces. The decomposition of the bundle $S^{2} T_{c}^{*}$ into irreducible $G$-invariant sub-bundles and the branching law of [18] then permit us to determine the multiplicities of the isotypic components of $C^{\infty}\left(S^{2} T_{C}^{*}\right.$ ) (Proposition 4.2). According to [11], $\Delta$ is equal to a constant multiple of the Casimir operator of the $G$-module $C^{\infty}\left(S^{2} T_{C}^{*}\right)$; in $\S 4$ we use this fact to derive properties of these isotypic components and of $\Delta$.

\section{Hermitian manifolds.}

Let $X$ be a complex manifold of complex dimension $n$ endowed with a Hermitian metric $g$. We denote by $J$ its complex structure, and by $T$ and $T^{*}$ its tangent and cotangent bundles. By $\otimes^{k} E, S^{l} E, \wedge^{j} E$, we shall mean the $k$-th tensor product, the $l$-th symmetric product and the $j$-th exterior product of a vector bundle $E$ over $X$, respectively. If $E$ is a vector bundle over $X$, we denote by $E_{C}$ its complexification, by $\mathscr{E}$ the sheaf of sections of $E$ over $X$ and by $C^{\infty}(E)$ the space of global sections of $E$ over $X$. Let $C^{\infty}(X)$ be the space of complex-valued functions on $X$. If $\alpha, \beta \in T_{C}^{*}$, we identify the symmetric product $\alpha \cdot \beta$ with the element $\alpha \otimes \beta+\beta \otimes \alpha$ of $\otimes^{2} T_{C}^{*}$.

Let $G$ be the sub-bundle of $\wedge^{2} T^{*} \otimes \wedge^{2} T^{*}$ consisting of those tensors satisfying the first Bianchi identity considered in $[4, \S 3]$. We denote by $G^{0}$ and $S_{0}^{2} T^{*}$ the sub-bundles of $G$ and $S^{2} T^{*}$ equal to the kernels of the trace mappings

$$
\operatorname{Tr}: G \rightarrow S^{2} T^{*}, \quad \operatorname{Tr}: S^{2} T^{*} \rightarrow \boldsymbol{R},
$$

defined by

$$
(\operatorname{Tr} u)(\xi, \eta)=\sum_{j=1}^{2 n} u\left(t_{j}, \xi, t_{j}, \eta\right), \quad \operatorname{Tr} h=\sum_{j=1}^{2 n} h\left(t_{j}, t_{j}\right),
$$

for $u \in G_{x}, h \in S^{2} T_{x}^{*}$, where $x \in X$ and $\left\{t_{1}, \ldots, t_{2 n}\right\}$ is an orthonormal basis of $T_{x}$.

The complex structure $J$ induces involutions

$$
J: \wedge^{2} T^{*} \rightarrow \wedge^{2} T^{*}, \quad J: S^{2} T^{*} \rightarrow \mathrm{S}^{2} T^{*},
$$


defined by

$$
\beta^{J}(\xi, \eta)=\beta(J \xi, J \eta), \quad h^{J}(\xi, \eta)=h(J \xi, J \eta)
$$

for $\beta \in \wedge^{2} T^{*}, h \in S^{2} T^{*}$ and $\xi, \eta \in T$. Then $G$ is stable under the involution

$$
J=J \otimes J: \wedge^{2} T^{*} \otimes \wedge^{2} T^{*} \rightarrow \wedge^{2} T^{*} \otimes \wedge^{2} T^{*} .
$$

We obtain the orthogonal decompositions

$$
\wedge^{2} T^{*}=T_{R}^{1,1} \oplus\left(\wedge^{2} T^{*}\right)^{-}, \quad S^{2} T^{*}=\left(S^{2} T^{*}\right)^{+} \oplus\left(S^{2} T^{*}\right)^{-}, \quad G=G^{+} \oplus G^{-}
$$

into direct sums of the eigenbundles $T_{\boldsymbol{R}}^{1,1},\left(\wedge^{2} T^{*}\right)^{-},\left(S^{2} T^{*}\right)^{+},\left(S^{2} T^{*}\right)^{-}, G^{+}$ and $G^{-}$corresponding to the eigenvalues +1 and -1 , respectively, of the involutions $J$. In fact, $T_{\boldsymbol{R}}^{1,1}$ is the bundle of real forms of type $(1,1)$ and $\left(S^{2} T^{*}\right)^{+}$is the bundle of Hermitian symmetric 2 -forms. We denote by $\pi_{+}$ and $\pi_{\text {- }}$ the orthogonal projections of $S^{2} T^{*}$ onto $\left(S^{2} T^{*}\right)^{+}$and $\left(S^{2} T^{*}\right)^{-}$, respectively. It is easily verified that

$$
\operatorname{Tr}\left(G^{+}\right) \subset\left(S^{2} T^{*}\right)^{+}, \quad \operatorname{Tr}\left(G^{-}\right) \subset\left(S^{2} T^{*}\right)^{-} .
$$

If $h$ is an element of $S^{2} T^{*}$, we consider the element $\breve{h}$ of $\otimes^{2} T^{*}$ defined by

$$
\breve{h}(\xi, \eta)=h(J \xi, \eta),
$$

for all $\xi, \eta \in T$. If $h \in\left(S^{2} T^{*}\right)^{+}$, then $\breve{h}$ is an element of $T_{R}^{1,1}$; on the other hand, if $h$ belongs to $\left(S^{2} T^{*}\right)^{-}$, so does $\breve{h}$. We thus obtain a canonical isomorphism

$$
\left(S^{2} T^{*}\right)^{+} \rightarrow T_{R}^{1,1},
$$

sending $h \in\left(S^{2} T^{*}\right)^{+}$into the form $\breve{h}$ of type $(1,1)$, and an endomorphism

$$
J:\left(S^{2} T^{*}\right)^{-} \rightarrow\left(S^{2} T^{*}\right)^{-},
$$

sending $h \in\left(S^{2} T^{*}\right)^{-}$into the symmetric 2 -form $J(h)=\breve{h}$, which satisfies $J^{2}=-$ id. The image of $g$ under the isomorphism (1.1) is equal to the Kähler form $\omega$ of $X$.

Let $T^{p, q}$ be the bundle of complex differential forms of type $(p, q)$ on $X$. The eigenbundles corresponding to the eigenvalues $+i$ and $-i$ of the endomorphism $J$ of $\left(S^{2} T^{*}\right)_{c}^{-}$are the bundles $S^{2} T^{1,0}$ and $S^{2} T^{0,1}$ of symmetric forms of type $(2,0)$ and $(0,2)$, respectively. Thus we have

$$
\left(T_{R}^{1,1}\right)_{C}=T^{1,1}, \quad\left(S^{2} T^{*}\right)_{\bar{c}}^{-}=S^{2} T^{1,0} \oplus S^{2} T^{0,1} .
$$


Let $\nabla$ be the Levi-Civita connection of the Riemannian manifold $X$. Let

$$
\operatorname{div}: S^{2} \mathscr{T}^{*} \rightarrow \mathscr{T}^{*}
$$

be the first-order differential operator defined by

$$
(\operatorname{div} h)(\xi)=-\sum_{j=1}^{2 n}(\nabla h)\left(t_{j}, t_{j}, \xi\right),
$$

for $h \in C^{\infty}\left(S^{2} T^{*}\right), \xi \in T_{x}$, where $x \in X$ and $\left\{t_{1}, \ldots, t_{2 n}\right\}$ is an orthonormal basis of $T_{x}$. Then we see that

$$
\text { (1.3) }(\operatorname{div} h)(\xi)=\left(d^{*} \breve{h}\right)(J \xi) \text {, }
$$

for all $h \in C^{\infty}\left(\left(S^{2} T^{*}\right)^{+}\right), \xi \in T$, where $d^{*}$ is the formal adjoint of the exterior derivative $d$. Moreover, the following lemma is easily verified.

LEMMA 1.1. Suppose that $g$ is a Kähler metric and let $f \in C^{\infty}(X)$. If $h$ is the section $\pi_{+}$Hess $f$ of $\left(S^{2} T^{*}\right)_{c}^{+}$, then we have

$$
\text { (1.4) } \quad \check{h}=i \partial \bar{\partial} f \text {. }
$$

\section{The complex quadric.}

Let $n$ be an integer $\geq 2$. We endow $C^{n+2}$ with its usual Hermitian scalar product

$$
\langle z, w\rangle=\sum_{j=0}^{n+1} z_{j} \bar{w}_{j},
$$

for $z=\left(z_{0}, z_{1}, \ldots, z_{n+1}\right), w=\left(w_{0}, w_{1}, \ldots, w_{n+1}\right) \in \boldsymbol{C}^{n+2}$, with the real scalar product

$$
\langle z, w\rangle_{R}=\operatorname{Re}\langle z, w\rangle,
$$

and with the complex bilinear form

$$
h(z, w)=\sum_{j=0}^{n+1} z_{j} w_{j}
$$

We consider the complex projective space $\boldsymbol{C P}^{n+1}$ of dimension $n+1$ endowed with the Fubini-Study metric $\widetilde{g}$ of constant holomorphic curvature 4. If

$$
\pi: \boldsymbol{C}^{n+2}-\{0\} \rightarrow \boldsymbol{C} \boldsymbol{P}^{n+1}
$$

is the natural projection, and if $S^{2 n+3}$ is the unit sphere of $C^{n+2}$ endowed with the Riemannian metric induced by the real scalar product (2.1), then 


$$
\pi: S^{2 n+3} \rightarrow \boldsymbol{C P}^{n+1}
$$

is a Riemannian submersion. In fact, the tangent space $T_{z}\left(S^{2 n+3}\right)$ of the sphere $S^{2 n+3}$ at $z \in S^{2 n+3}$ is identified with the space

$$
\left\{(z, u) \mid u \in C^{n+2},\langle z, u\rangle_{R}=0\right\} .
$$

We also consider its subspace

$$
H_{z}\left(S^{2 n+3}\right)=\left\{(z, u) \mid u \in C^{n+2},\langle z, u\rangle=0\right\} ;
$$

if $u \in C^{n+2}$ satisfies $\langle z, u\rangle=0$, we shall sometimes write $u$ for the element $(z, u)$ of $H_{z}\left(S^{2 n+3}\right)$. If $T_{\pi(z)}\left(\boldsymbol{C P}^{n+1}\right)$ is the tangent space of $\boldsymbol{C P}^{n+1}$ at $\pi(z)$, then

$$
\pi_{*}: H_{z}\left(S^{2 n+3}\right) \rightarrow T_{\pi(z)}\left(\boldsymbol{C P}^{n+1}\right)
$$

is an isometry. Moreover, if $u \in C^{n+2}$ satisfies $\langle z, u\rangle=0$ and $\lambda$ is an element of $\boldsymbol{C}$, with $|\lambda|=1$, then $(\lambda z, \lambda u)$ belongs to $H_{\lambda z}\left(S^{2 n+3}\right)$ and

$$
\pi_{*}(z, u)=\pi_{*}(\lambda z, \lambda u) .
$$

We henceforth suppose that $X$ is the complex quadric $Q_{n}$, which is the complex hypersurface of complex projective space $\boldsymbol{C P}^{n+1}$ defined by the homogeneous equation

$$
\zeta_{0}^{2}+\zeta_{1}^{2}+\cdots+\zeta_{n+1}^{2}=0
$$

where $\zeta_{0}, \zeta_{1}, \ldots, \zeta_{n+1}$ are the standard complex coordinates of $\boldsymbol{C}^{n+2}$. Let $g$ be the Kähler metric on $X$ induced by the metric $\tilde{g}$ of $\boldsymbol{C P}^{n+1}$. In fact, we have

$$
\begin{aligned}
Q_{n} & =\left\{\pi(z) \mid z \in C^{n+2}-\{0\}, h(z, z)=0\right\}, \\
& =\left\{\pi(z) \mid z \in S^{2 n+3}-\{0\}, h(z, z)=0\right\} .
\end{aligned}
$$

If $z \in S^{2 n+3}$ satisfies $h(z, z)=0$, we consider the subspace

$$
H_{z}^{\prime}\left(S^{2 n+3}\right)=\left\{(z, u) \mid u \in C^{n+2},\langle z, u\rangle=0, h(z, u)=0\right\}
$$

of $H_{z}\left(S^{2 n+3}\right)$; then

$$
\pi_{*}: H_{z}^{\prime}\left(S^{2 n+3}\right) \rightarrow T_{\pi(z)}
$$

is an isometry (see [1], [10]).

Let $\left\{e_{0}, e_{1}, \ldots, e_{n+1}\right\}$ be the standard basis of $C^{n+2}$. Let $b$ be the point $\left(e_{0}+i e_{1}\right) / \sqrt{2}$ of $S^{2 n+3}$; then $h(b, b)=0$ and $a=\pi(b)$ is a point of $Q_{n}$. We consider the vectors 


$$
\tilde{\nu}=\left(i e_{0}+e_{1}\right) / \sqrt{2}, \quad \tilde{\nu}^{\prime}=\left(-e_{0}+i e_{1}\right) / \sqrt{2}
$$

of $H_{b}\left(S^{2 n+3}\right)$; clearly, we have $\pi_{*} \widetilde{\nu}^{\prime}=J \pi_{*} \widetilde{\nu}$. Then

$$
\left\{e_{2}, \ldots, e_{n+1}, i e_{2}, \ldots, i e_{n+1}\right\}
$$

is an orthonormal basis of $H_{b}^{\prime}\left(S^{2 n+3}\right)$ and $\left\{\tilde{\nu}, \tilde{\nu}^{\prime}\right\}$ is an orthonormal basis for the orthogonal complement of $H_{b}^{\prime}\left(S^{2 n+3}\right)$ in $H_{b}\left(S^{2 n+3}\right)$.

The group $S U(n+2)$ acts on $\boldsymbol{C}^{n+2}$ and $\boldsymbol{C P}^{n+1}$ by holomorphic isometries; its subgroup $S O(n+2)$ leaves the submanifold $X$ of $\boldsymbol{C P}^{n+1}$ invariant, and acts transitively on $X$ by holomorphic isometries. The isotropy group of the point $a$ is equal to the subgroup $H=S O(2) \times S O(n)$ of $S O(n+2)$ consisting of the matrices

$$
\left(\begin{array}{cc}
A & 0 \\
0 & B
\end{array}\right)
$$

where $A \in S O(2)$ and $B \in S O(n)$. For $\theta \in \boldsymbol{R}$, we denote by $R(\theta)$ the element

$$
\left(\begin{array}{cc}
\cos \theta & -\sin \theta \\
\sin \theta & \cos \theta
\end{array}\right)
$$

of $S O(2)$ and by $R^{\prime}(\theta)$ the element

$$
\left(\begin{array}{cc}
R(\theta) & 0 \\
0 & I
\end{array}\right)
$$

of $H$, where $I$ is the identity element of $S O(n)$. Since

$$
R^{\prime}(\theta) b=e^{-i \theta} b, \quad R^{\prime}(\theta)_{*}\left(b, i e_{0}+e_{1}\right)=\left(e^{-i \theta} b, e^{i \theta}\left(i e_{0}+e_{1}\right)\right),
$$

we see that

$$
\begin{aligned}
R^{\prime}(\theta)_{*} \pi_{*} \tilde{\nu} & =\pi_{*}\left(e^{-i \theta} b, e^{i \theta}\left(i e_{0}+e_{1}\right) / \sqrt{2}\right) \\
& =\pi_{*}\left(b, e^{i 2 \theta}\left(i e_{0}+e_{1}\right) / \sqrt{2}\right) \\
& =\cos 2 \theta \cdot \pi_{*} \tilde{\nu}+\sin 2 \theta \cdot J \pi_{*} \tilde{\nu}
\end{aligned}
$$

and

$$
R^{\prime}(\theta)_{*} \pi_{*} e_{j}=\pi_{*}\left(e^{-i \theta} b, e_{j}\right)=\pi_{*}\left(b, e^{i \theta} e_{j}\right),
$$

for $2 \leq j \leq n+1$.

Let $\left\{e_{1}^{\prime}, \ldots, e_{n}^{\prime}\right\}$ be the standard basis of $\boldsymbol{C}^{n}$ and let

$$
\psi: T_{a} \rightarrow \boldsymbol{C}^{n}
$$

be the isomorphism of real vector spaces determined by 


$$
\psi \pi_{*} e_{j}=e_{j-1}^{\prime}, \quad \psi \pi_{*} i e_{j}=i e_{j-1}^{\prime},
$$

for $2 \leq j \leq n+1$. If we identify $T_{a}$ with $C^{n}$ by means of this isomorphism $\psi$, since $J \pi_{*} e_{j}=\pi_{*} i e_{j}$, for $2 \leq j \leq n+1$, the complex structure of $T_{a}$ is the one determined by the multiplication by $i$ on $\boldsymbol{C}^{n}$, and the Kähler metric $g$ at $a$ is the one obtained from the standard Hermitian scalar product of $\boldsymbol{C}^{n}$. Moreover, by (2.3) we see that the action of the element

$$
\phi=\left(\begin{array}{cc}
R(\theta) & 0 \\
0 & B
\end{array}\right)
$$

of $H$, with $B \in S O(n), \theta \in \boldsymbol{R}$, on $T_{a}=\boldsymbol{C}^{n}$ is given by

$$
\phi_{*} \zeta=e^{i \theta} B \zeta,
$$

for $\zeta \in C^{n}$, where $S O(n)$ is considered as a subgroup of $S U(n)$. In particular, if $\phi$ is the element $R^{\prime}(\theta)$ of $H$, we have

$$
R^{\prime}(\theta)_{*} \zeta=\cos \theta \cdot \zeta+\sin \theta \cdot J \zeta
$$

for $\zeta \in T_{a}$. Moreover, if $\mu$ is a unit vector of $T_{a}\left(\boldsymbol{C P}^{n+1}\right)$ normal to $X$, from (2.2) it follows that

$$
R^{\prime}(\theta)_{*} \mu=\cos 2 \theta \cdot \mu+\sin 2 \theta \cdot J \mu .
$$

If $j=R^{\prime}(\pi / 2)$, the element $s=j^{2}$ of $S O(n+2)$ determines an involution $\sigma$ of $S O(n+2)$ which sends $\phi \in S O(n+2)$ into $s \phi s^{-1}$. Then $H$ is equal to the identity component of the set of fixed points of $\sigma$, and $(G, H)$ is a Riemannian symmetric pair. The corresponding Cartan decomposition of the Lie algebra $g$ of $S O(n+2)$ is

$$
\mathfrak{g}=\mathfrak{h} \oplus \mathfrak{m},
$$

where $\mathfrak{h}$ is the Lie algebra of $H$ and $\mathfrak{m}$ is the space of all matrices

$$
\left(\begin{array}{ccc}
0 & 0 & { }^{-t} \xi \\
0 & 0 & -{ }^{-t} \eta \\
\xi & \eta & 0
\end{array}\right)
$$

of $\mathrm{g}$, where $\xi, \eta$ are vectors of $\boldsymbol{R}^{n}$ considered as column vectors. We identify $m$ with the tangent space of $Y=S O(n+2) / H$ at the coset of the identity element of $S O(n+2)$, and the vector $(\xi, \eta) \in \boldsymbol{R}^{n} \oplus \boldsymbol{R}^{n}$ with the matrix (2.8) of $\mathrm{m}$.

Since $S O(n+2)$ acts transitively on $X$, we have a diffeomorphism $\Psi$ : $Y \rightarrow X$, sending $\phi \cdot H$ into $\phi(a)$, for $\phi \in S O(n+2)$. The restriction of $\operatorname{Ad} j$ to $\mathfrak{m}$ is a complex structure on $\mathfrak{m}$, and so gives rise to an $S O(n+2)$ - 
invariant complex structure on $Y$. If $B$ is the Killing form of $\mathfrak{g}$, let $g_{0}$ be the unique $S O(n+2)$-invariant metric on $Y$ whose restriction to $m$ is equal to $-B$. Endowed with this complex structure and the metric $g_{0}$, the manifold $Y$ is a Hermitian symmetric space. It is easily verified that the isomorphism $\Psi_{*}: \mathfrak{m} \rightarrow T_{a}$ sends $(\xi, \eta) \in \mathfrak{m}$, with $\xi, \eta \in \boldsymbol{R}^{n}$, into $(\xi+i \eta) / \sqrt{2}$ $\in C^{n}$. Hence we see that $\Psi_{*} \circ \operatorname{Ad} j=J \circ \Psi_{*}$ and that $g_{0}=4 n \Psi^{*} g$. Thus $\Psi$ is a holomorphic isometry from the Hermitian symmetric space $Y$, endowed with the metric $1 / 4 n \cdot g_{0}$, to $X$; henceforth, we shall identify these two Kähler manifolds by means of this isometry (see [10]). From Proposition 9.7, Chapter XI of [10], it follows that $X$ is an Einstein manifold and that its Ricci tensor Ric is given by

(2.9) $\quad$ Ric $=2 n g$.

We now recall some of the results of [15] (see also [7]). The second fundamental form $B$ of the complex hypersurface $X$ of $\boldsymbol{C P}^{n+1}$ is a symmetric 2-form with values in the normal bundle of $X$ in $\boldsymbol{C P}^{n+1}$. We denote by $S$ the bundle of unit vectors of this normal bundle. Let $x \in X$ and $\nu \in S_{x}$. We consider the element $h_{\nu}$ of $S^{2} T_{x}^{*}$ defined by

$$
h_{\nu}(\xi, \eta)=\widetilde{g}(B(\xi, \eta), \nu)
$$

for all $\xi, \eta \in T_{x}$. By means of the metric $g$, we identify the 2-form $h_{\nu}$ with a symmetric endomorphism $K_{\nu}$ of $T_{x}$. If $\mu$ is another element of $S_{x}$, we have

$$
\mu=\cos 2 \theta \cdot \nu+\sin 2 \theta \cdot J \nu,
$$

with $\theta \in \boldsymbol{R}$. Then it is easily verified that

$$
K_{\mu}=\cos 2 \theta \cdot K_{\nu}+\sin 2 \theta \cdot J K_{\nu} .
$$

In particular, we have

$$
K_{J \nu}=J K_{\nu} .
$$

Since our manifolds are Kähler, we see that

(2.12) $J K_{\nu}=-K_{\nu} J$,

and, by (2.9), that $K_{\nu}$ is an involution (see [15]). We call $K_{\nu}$ the real structure of the quadric associated to the unit normal $\nu$. From (2.12), it follows that $h_{\nu}$ belongs to $\left(S^{2} T^{*}\right)^{-}$and that

(2.13) $\quad \check{h}_{\nu}=-h_{J \nu}$.

We denote by $T_{\nu, x}^{+}, T_{\nu, x}^{-}$the eigenspaces of $K_{\nu}$, with eigenvalue 
equal to +1 and -1 , respectively. We recall that $J$ induces an isomorphism of $T_{\nu, x}^{+}$onto $T_{\nu, x}^{-}$and that

$$
T_{x}=T_{\nu, x}^{+} \oplus T_{\nu, x}^{-}
$$

is an orthogonal decomposition. If $\mu$ is the unit normal given be (2.10), then according to (2.11), we easily see that

$$
T_{\mu, x}^{+}=\left\{\cos \theta \cdot \xi+\sin \theta \cdot J \xi \mid \xi \in T_{\nu, x}^{+}\right\} .
$$

Since an element $\phi$ of $S O(n+2)$ acts on $\boldsymbol{C P}^{n+1}$ and $X$ by holomorphic isometries, we have

$$
B\left(\phi_{*} \xi, \phi_{*} \eta\right)=\phi_{*} B(\xi, \eta),
$$

for all $\xi, \eta \in T$. Thus, if $\mu$ is the tangent vector $\phi_{* \nu}$ belonging to $S_{\phi(x)}$, we see that

$$
h_{\mu}\left(\phi_{*} \xi, \phi_{*} \eta\right)=h_{\nu}(\xi, \eta)
$$

for all $\xi, \eta \in T_{x}$, and hence that

$$
K_{\mu} \phi_{*}=\phi_{*} K_{\nu}
$$

on $T_{x}$. Therefore $\phi$ induces isomorphisms

$$
\phi_{*}: T_{\nu, x}^{+} \rightarrow T_{\mu, \phi(x)}^{+}, \quad \phi_{*}: T_{\nu, x}^{-} \rightarrow T_{\mu, \phi(x) .}^{-}
$$

We say that the real structures of $X$ are oriented if, for all $x \in X$ and $\nu \in S_{x}$, the subspace $T_{\nu, x}^{+}$of $T_{x}$ is oriented in such a way that, for all $\phi \in$ $S O(n+2)$, the isomorphism

$$
\phi_{*}: T_{\nu, x}^{+} \rightarrow T_{\phi_{*} \nu, \phi(x)}^{+}
$$

is orientation-preserving.

We shall require the following lemma in $\S 5$. Let $q \geq 0$ and $0 \leq m_{1} \leq m_{2}$ $\leq \cdots \leq m_{2 q}=m$ be integers, with $m \geq 1$; we set $m_{0}=0$.

LEMMA 2.1. Let $x \in X$ and $u$ be an element of $\otimes^{m} T_{x}^{*}$. Suppose that, for any element $\nu$ of $S_{x}$ and for any orthonormal set $\left\{\xi_{1}, \ldots, \xi_{q}\right\}$ of elements of $T_{\nu, x}^{+}$, we have

$$
u\left(\eta_{1}, \ldots, \eta_{m}\right)=0,
$$

where

$$
\begin{aligned}
& \eta_{j}=\xi_{i}, \text { for } m_{i-1}+1 \leq j \leq m_{i}, \quad 1 \leq i \leq q, \\
& \eta_{j}=J \xi_{i} \text {, for } m_{i+q-1}+1 \leq j \leq m_{i+q}, 1 \leq i \leq q \text {. }
\end{aligned}
$$


Then, for any element $\nu$ of $S_{x}$ and for any orthonormal set $\left\{\xi_{1}, \ldots, \xi_{q}\right\}$ of elements of $T_{\nu, x}^{+}$, we have

$$
\begin{aligned}
& u\left(J \eta_{1}, \ldots, J \eta_{m}\right)=0, \\
& u\left(\eta_{1}+s J \eta_{1}, \ldots, \eta_{m}+s J \eta_{m}\right)=0,
\end{aligned}
$$

for all $s \in \boldsymbol{R}$, if $\eta_{1}, \ldots, \eta_{m}$ are the vectors of $T_{x}$ defined by (2.16).

PROOF : Let $\nu$ be an element of $S_{x}$ and $\left\{\xi_{1}, \ldots, \xi_{q}\right\}$ be an orthonormal set of elements of $T_{\nu, x}^{+}$. Let $\mu$ be the element (2.10) of $S_{x}$, with $\theta \in$ $\boldsymbol{R}$; then, by (2.15)

$$
\xi_{j}^{\prime}=\cos \theta \cdot \xi_{j}+\sin \theta \cdot J \xi_{j}
$$

belongs to $T_{\mu, x}^{+}$, for $1 \leq j \leq q$. By considering the orthonormal set $\left\{\xi_{1}^{\prime}, \ldots\right.$, $\left.\xi_{q}^{\prime}\right\}$ of elements of $T_{\mu, x}^{+}$, from our hypothesis we deduce the equality

$$
u\left(\cos \theta \cdot \eta_{1}+\sin \theta \cdot J \eta_{1}, \ldots, \cos \theta \cdot \eta_{m}+\sin \theta \cdot J \eta_{m}\right)=0,
$$

where $\eta_{1}, \ldots, \eta_{m}$ are the vectors of $T_{x}$ defined by (2.16). If we take $\theta=$ $\pi / 2$ in the above equality, we obtain the first of the desired relations. We write $f(\theta)=\tan \theta$. If $\theta \neq \pi / 2 \bmod \pi$, then (2.17) holds with $s=f(\theta)$. Since $f^{\prime}(0) \neq 0$, the relation (2.17) is valid for all $s \in \boldsymbol{R}$ in a neighborhood of 0 . However, the left-hand side of (2.17) is a polynomial in $s$ of degree $m$; therefore it vanishes identically.

Let $f$ be a complex-valued function on $C^{n+2}$, whose restriction to the unit sphere $S^{2 n+3}$ is invariant under $U(1)$. The restriction of $f$ to $S^{2 n+3}$ induces by passage to the quotient a function on $\boldsymbol{C P}^{n+1}$, whose restriction to $X$ we denote by $\tilde{f}$. For $r, s \geq 0$, we consider the $U(1)$-invariant function

$$
\begin{aligned}
f_{r, s}(\zeta)=\left(\left(\zeta_{0}+i \zeta_{1}\right)\left(\bar{\zeta}_{2}+i \bar{\zeta}_{3}\right)\right. & \\
& \left.-\left(\zeta_{2}+i \zeta_{3}\right)\left(\bar{\zeta}_{0}+i \bar{\zeta}_{1}\right)\right)^{s}\left(\zeta_{0}+i \zeta_{1}\right)^{r}\left(\bar{\zeta}_{0}+i \bar{\zeta}_{1}\right)^{r}
\end{aligned}
$$

on $\boldsymbol{C}^{n+2}$ and the function $\tilde{f}_{r, s}$ it induces on $X$. Consider the open subset

$$
V=\pi\left(\left\{\left(\zeta_{0}, \ldots, \zeta_{n+1}\right) \in C^{n+2} \mid \zeta_{0} \neq 0\right\}\right)
$$

of $\boldsymbol{C} \boldsymbol{P}^{n+1}$. We denote by $z=\left(z_{1}, \ldots, z_{n+1}\right)$ the holomorphic coordinate on $V$, where $z_{j}$ is the function which satisfies $\pi^{*} z_{j}=\zeta_{j} / \zeta_{0}$ on $C^{*} \times C^{n+1}$. We write

$$
z_{j}=x_{j}+i y_{j},
$$

for $1 \leq j \leq n+1$, where $x_{j}$ and $y_{j}$ are real-valued functions on $V$. Then we have 


$$
X \cap V=\left\{z \in V \mid 1+\sum_{j=1}^{n+1} z_{j}^{2}=0\right\}
$$

and the point $a$ belongs to $V$. We set

$$
\begin{array}{ll}
\xi_{1}=2\left(\frac{\partial}{\partial x_{1}}\right)_{a}, & \eta_{1}=2\left(\frac{\partial}{\partial y_{1}}\right)_{a}, \\
\xi_{j}=\sqrt{2}\left(\frac{\partial}{\partial x_{j}}\right)_{a}, & \eta_{j}=\sqrt{2}\left(\frac{\partial}{\partial y_{j}}\right)_{a},
\end{array}
$$

for $2 \leq j \leq n+1$. Then the mapping $\pi_{*}: H_{b}\left(S^{2 n+3}\right) \rightarrow T_{a}\left(\boldsymbol{C P} \boldsymbol{P}^{n+1}\right)$ is determined by

$$
\begin{array}{ll}
\pi_{*} \tilde{\nu}=\xi_{1}, & \pi_{*} \tilde{\nu}^{\prime}=\eta_{1}, \\
\pi_{*} e_{j}=\xi_{j}, & \pi_{*} i e_{j}=\eta_{j},
\end{array}
$$

for $2 \leq j \leq n+1$. Thus $\left\{\xi_{2}, \ldots, \xi_{n+1}, \eta_{2}, \ldots, \eta_{n+1}\right\}$ is an orthonormal basis of $T_{a}$ and the element $\nu^{\prime}=\eta_{1}$ of $S_{a}$ satisfies $J \nu^{\prime}=-\xi_{1}$. Since the complex vector fields $\partial / \partial z_{j}-z_{j} / z_{1} \partial / \partial z_{1}$ are tangent to $X$ on a neighborhood of $a$ for $2 \leq j \leq n+1$, we easily verify that the second fundamental form $B$ of $X$ is given at the point $a$ by

$$
\begin{aligned}
& B\left(\xi_{j}, \xi_{k}\right)=\delta_{j k} \nu^{\prime}=-B\left(\eta_{j}, \eta_{k}\right), \\
& B\left(\xi_{j}, \eta_{k}\right)=\delta_{j k} J \nu^{\prime},
\end{aligned}
$$

for $2 \leq j \leq n+1$. We thus obtain

$$
K_{\nu^{\prime}} \xi_{j}=\xi_{j}, \quad K_{\nu^{\prime}} \eta_{j}=-\eta_{j},
$$

for $2 \leq j \leq n+1$. Therefore $\left\{\xi_{2}, \ldots, \xi_{n+1}\right\}$ is an orthonormal basis of $T_{\nu^{\prime}, a}^{+}$, while $\left\{\eta_{2}, \ldots, \eta_{n+1}\right\}$ is an orthonormal basis of $T_{\nu^{\prime}, a}^{-}$.

By (2.7), we see that the element (2.4) of $H$, with $\theta \in \boldsymbol{R}, B \in S O(n)$, satisfies $\phi_{*} \nu^{\prime}=\nu^{\prime}$ if and only if $\theta=0 \bmod \pi$. Let $\left\{\tilde{e}_{1}, \ldots, \tilde{e}_{n}\right\}$ be the standard basis of $\boldsymbol{R}^{n}$. We identify $T_{\nu^{\prime}, a}^{+}$with $\boldsymbol{R}^{n}$ by means of the isomorphism $\boldsymbol{R}^{n} \rightarrow T_{\nu^{\prime}, a}^{+}$sending $\tilde{e}_{j}$ into $\xi_{j+1}$, for $1 \leq j \leq n$. When $\theta=0 \bmod \pi$, by (2.5) and (2.18), the action of the element (2.4) of $H$ on $T_{\nu^{\prime}, a}^{+}=\boldsymbol{R}^{n}$ is given by

$$
\phi_{*} u= \begin{cases}B u, & \text { if } \theta=0 \bmod 2 \pi, \\ -B u, & \text { if } \theta=\pi \bmod 2 \pi,\end{cases}
$$

for $u \in \boldsymbol{R}^{n}$, where $B$ acts on $\boldsymbol{R}^{n}$ according to the natural representation of $S O(n)$. Therefore, if $n$ is even, for $\theta=0 \bmod \pi$ the action of $\phi_{*}$ on $T_{\nu^{\prime}, a}^{+}$ is always orientation-preserving.

According to (2.7), the action of $H$ on $S$ is transitive. Thus, if $n$ is even, we see that an orientation of the real vector space $T_{\nu^{\prime}, a}^{+}$determines 
an orientation of all the real structures of $X$. We also remark that, if $n$ is odd, the real structures of $X$ are not orientable.

We take this opportunity to point out that the definitions of the vectors $\xi_{j}, \eta_{j}, \xi_{n+1}, \eta_{n+1}$, with $1 \leq j \leq n$, of $\S 6$ of [7] should read as follows :

$$
\begin{array}{ll}
\xi_{j}=\sqrt{2}\left(\frac{\partial}{\partial x_{j}}\right)_{a}, & \eta_{j}=\sqrt{2}\left(\frac{\partial}{\partial y_{j}}\right)_{a}, \\
\xi_{n+1}=2\left(\frac{\partial}{\partial x_{n+1}}\right)_{a}, & \eta_{n+1}=2\left(\frac{\partial}{\partial y_{n+1}}\right)_{a} .
\end{array}
$$

Moreover, with these definitions, if $\xi=\left(\xi_{j}+\eta_{j}\right) / \sqrt{2}, \eta=\left(\xi_{k}+\eta_{k}\right) / \sqrt{2}$ for $1 \leq j<k \leq n$, we have

$$
\xi+J \eta=v_{j k}(a), \quad \eta+J \xi=v_{k j}(a) .
$$

\section{Symmetric 2 -forms on the quadric.}

Let $x \in X$ and $\nu$ be an element of $S_{x}$. For $\beta \in T_{\boldsymbol{R}, x}^{1,1}$ and $h \in\left(S^{2} T^{*}\right)_{x}^{+}$, we define elements $K_{\nu}(\beta)$ of $\wedge^{2} T_{x}^{*}$ and $K_{\nu}(h)$ of $S^{2} T_{x}^{*}$ by

$$
K_{\nu}(\beta)(\xi, \eta)=\beta\left(K_{\nu} \xi, K_{\nu} \eta\right), \quad K_{\nu}(h)(\xi, \eta)=h\left(K_{\nu} \xi, K_{\nu} \eta\right),
$$

for all $\xi, \eta \in T_{x}$. Using (2.12), we see that $K_{\nu}(\beta)$ and $K_{\nu}(h)$ belong to $T_{\boldsymbol{R}}^{1,1}$ and $\left(S^{2} T^{*}\right)^{+}$, respectively. By $(2.11)$, we also observe that $K_{\nu}(\beta)$ and $K_{\nu}(h)$ do not depend on the choice of the unit normal $\nu$. We thus obtain canonical involutions of $T_{\boldsymbol{R}}^{1,1}$ and $\left(S^{2} T^{*}\right)^{+}$over all of $X$, which give us decompositions

$$
\begin{aligned}
& T_{R}^{1,1}=\left(T_{R}^{1,1}\right)^{+} \oplus\left(T_{R}^{1,1}\right)^{-}, \\
& \left(S^{2} T^{*}\right)^{+}=\left(S^{2} T^{*}\right)^{++} \oplus\left(S^{2} T^{*}\right)^{+-}
\end{aligned}
$$

into the direct sums of the eigenbundles $\left(T_{\boldsymbol{R}}^{1,1}\right)^{+},\left(T_{\boldsymbol{R}}^{1,1}\right)^{-},\left(S^{2} T^{*}\right)^{++}$and $\left(S^{2} T^{*}\right)^{+-}$corresponding to the eigenvalues +1 and -1 , respectively, of these involutions. By (2.12), we see that the mapping (1.1) induces by restriction isomorphisms

$$
\left(S^{2} T^{*}\right)^{+-} \rightarrow\left(T_{R}^{1,1}\right)^{+}, \quad\left(S^{2} T^{*}\right)^{++} \rightarrow\left(T_{R}^{1,1}\right)^{-} .
$$

The metric $g$ is a section of $\left(S^{2} T^{*}\right)^{++}$and generates a line bundle $\{g\}$, whose orthogonal complement in $\left(S^{2} T^{*}\right)^{++}$is the sub-bundle

$$
\left(S^{2} T^{*}\right)_{0}^{++}=\left(S_{0}^{2} T^{*}\right) \cap\left(S^{2} T^{*}\right)^{++}
$$

of $\left(S^{2} T^{*}\right)^{++}$consisting of the forms with zero-trace.

We easily see that 


$$
\begin{aligned}
& \left(T_{R}^{1,1}\right)_{x}^{+}=\left\{\beta \in T_{R, x}^{1,1} \mid \beta(\xi, J \eta)=0 \text { for all } \xi, \eta \in T_{\nu, x}^{+}\right\} \\
& \left(S^{2} T^{*}\right)_{x}^{+-}=\left\{h \in\left(S^{2} T^{*}\right)_{x}^{+} \mid h(\xi, \eta)=0 \text { for all } \xi, \eta \in T_{\nu, x}^{+}\right\}
\end{aligned}
$$

We thus obtain an isomorphism

$$
\rho_{\nu}:\left(T_{R}^{1,1}\right)_{x}^{+} \rightarrow \wedge^{2} T_{\nu, x}^{+*},
$$

sending an element of $\left(T_{R}^{1,1}\right)_{x}^{+}$into its restriction to $\wedge^{2} T_{\nu, x}^{+}$.

The sub-bundle $E$ of $\left(S^{2} T^{*}\right)^{-}$of rank 2, whose fiber at $x \in X$ is the subspace of $\left(S^{2} T^{*}\right)_{x}^{-}$generated by $h_{\nu}$ and $h_{J \nu}$, where $\nu$ is an element of $S_{x}$, is well-defined. If we denote by $\left(S^{2} T^{*}\right)^{-\perp}$ the orthogonal complement of $E$ in $\left(S^{2} T^{*}\right)^{-}$, we obtain the orthogonal decomposition

$$
S^{2} T^{*}=E \oplus\left(S^{2} T^{*}\right)^{-\perp} \oplus\{g\} \oplus\left(S^{2} T^{*}\right)_{0}^{++} \oplus\left(S^{2} T^{*}\right)^{+-} .
$$

By (2.13), we see that $E$ is stable under the endomorphism (1.2) of $\left(S^{2} T^{*}\right)^{-}$; since $J: T \rightarrow T$ is an isometry, the orthogonal complement $\left(S^{2} T^{*}\right)^{-\perp}$ of $E$ is also stable under this endomorphism. We denote by $E^{\prime}$, $E^{\prime \prime},\left(S^{2} T^{1,0}\right)^{\perp},\left(S^{2} T^{0,1}\right)^{\perp}$ the eigenbundles corresponding to the eigenvalues $+i$ and $-i$ of the endomorphisms $J$ of $E_{C}$ and $\left(S^{2} T^{*}\right)_{c}^{-\perp}$, respectively. In fact, by (2.13) we infer that $h_{\nu}+i h_{J \nu}$ generates $E_{x}^{\prime}$ and that $h_{\nu}-i h_{J \nu}$ is a generator of $E_{x}^{\prime \prime}$. Clearly, we have

$$
E^{\prime \prime}=\overline{E^{\prime}}, \quad\left(S^{2} T^{0,1}\right)^{\perp}=\overline{\left(S^{2} T^{1,0}\right)^{\perp}} .
$$

We obtain the orthogonal decompositions

$$
\begin{aligned}
& S^{2} T^{1,0}=E^{\prime} \oplus\left(S^{2} T^{1,0}\right)^{\perp}, \quad S^{2} T^{0,1}=E^{\prime \prime} \oplus\left(S^{2} T^{0,1}\right)^{\perp} \\
& S^{2} T_{C}^{*}=E^{\prime} \oplus E^{\prime \prime} \oplus\left(S^{2} T^{1,0}\right)^{\perp} \oplus\left(S^{2} T^{0,1}\right)^{\perp} \\
& \\
& \oplus\{g\}_{C} \oplus\left(S^{2} T^{*}\right)_{0 C}^{++} \oplus\left(S^{2} T^{*}\right)_{C}^{+-}
\end{aligned}
$$

We consider the holomorphic coordinate $z=\left(z_{1}, \ldots, z_{n+1}\right)$ of $\S 2$ on the open subset $V$ of $\boldsymbol{C P}^{n+1}$, and the holomorphic coordinate $w=\left(w_{1}, \ldots, w_{n}\right)$ on a neighborhood of $a$ in $X$, where $w_{j}$ is the restriction of the function $z_{j+1}$ to $X \cap V$, for $1 \leq j \leq n$. If $\nu^{\prime}$ is the unit normal $\pi_{*} \widetilde{\nu}^{\prime} \in S_{a}$ of $\S 2$, it is easily verified that

$$
h_{\nu^{\prime}}+i h_{J^{\prime}}=\frac{1}{4} \sum_{j=1}^{n}\left(d w_{j} \cdot d w_{j}\right)(a), \quad g(a)=\frac{1}{4} \sum_{j=1}^{n}\left(d w_{j} \cdot d \bar{w}_{j}\right)(a)
$$

and that

$$
\left(S^{2} T^{1,0}\right)_{a}^{\perp}=\left\{\sum_{j, k=1}^{n} c_{j k}\left(d w_{j} \cdot d w_{k}\right)(a) \mid c_{j k}=c_{k j} \in C, \sum_{j=1}^{n} c_{j j}=0\right\},
$$




$$
\begin{aligned}
& \left(S^{2} T^{*}\right)_{0 C, a}^{++}=\left\{\sum_{j, k=1}^{n} c_{j k}\left(d w_{j} \cdot d \bar{w}_{k}\right)(a) \mid c_{j k}=c_{k j} \in \boldsymbol{C}, \sum_{j=1}^{n} c_{j j}=0\right\}, \\
& \left(S^{2} T^{*}\right)_{C, a}^{+-}=\left\{\sum_{j, k=1}^{n} c_{j k}\left(d w_{j} \cdot d \bar{w}_{k}\right)(a) \mid c_{j k}=-c_{k j} \in C\right\} .
\end{aligned}
$$

We now suppose that $X$ is the quadric $Q_{4}$ of complex dimension 4 . We choose an orientation of the real structures of $X$. We define an involution

$$
*:\left(T_{\boldsymbol{R}}^{1,1}\right)^{+} \rightarrow\left(T_{\boldsymbol{R}}^{1,1}\right)^{+}
$$

as follows. Let $x \in X$ and $\nu$ be an element of $S_{x}$. We have an involution * of $\wedge^{2} T_{\nu, x}^{+*}$ defined in terms of the orientation of the 4-dimensional space $T_{\nu, x}^{+}$and its metric. Then the involution

$$
*:\left(T_{R}^{1,1}\right)_{x}^{+} \rightarrow\left(T_{R}^{1,1}\right)_{x}^{+}
$$

is the unique mapping which makes the diagram

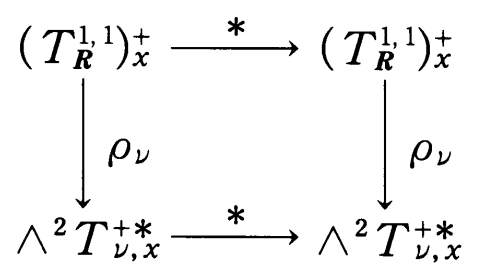

commutative. We now show that the mapping (3.5) is independent of the choice of the unit normal $\nu$ at $x$. Since the group $S O(n+2)$ acts on the spaces $T_{\nu, x}^{+}$by orientation-preserving isometries, it suffices to verify this fact when $x=a$. In this case, if $\nu, \mu$ are elements of $S_{a}$ related by (2.10), with $\theta \in \boldsymbol{R}$, then, because of (2.6) and (3.1), the diagram

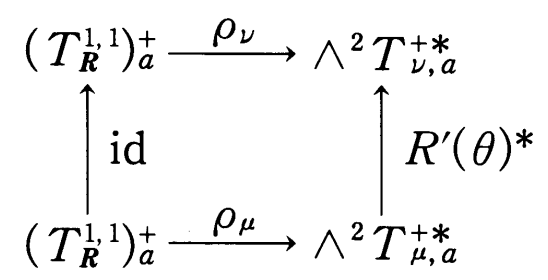

is easily seen to commute. Thus, since $R^{\prime}(\theta)_{*}: T_{\nu, a}^{+} \rightarrow T_{\mu, a}^{+}$is an isometry, which preserves the orientations, the two mappings (3.5) defined in terms of $\nu$ and $\mu$ are equal.

From this involution of $\left(T_{R}^{1,1}\right)^{+}$, by means of the isomorphism (1.1), we obtain an involution of $\left(S^{2} T^{*}\right)^{+-}$, which we also denote by $*$ and which is easily seen to be an isometry. If $h \in\left(S^{2} T^{*}\right)_{x}^{+-}$and $\left\{\zeta_{1}, \zeta_{2}, \zeta_{3}, \zeta_{4}\right\}$ is an oriented orthonormal basis of $T_{\nu, x}^{+}$, then 


$$
(* h)\left(\zeta_{1}, J \zeta_{2}\right)=h\left(\zeta_{3}, J \zeta_{4}\right)
$$

If $F^{+}, F^{-}$are the eigenbundles corresponding to the eigenvalues +1 and -1 , respectively, of this involution of $\left(S^{2} T^{*}\right)^{+-}$, we obtain the orthogonal decompositions

$$
\begin{gathered}
S^{2} T^{*}=E \oplus\left(S^{2} T^{*}\right)^{-\perp} \oplus\{g\} \oplus\left(S^{2} T^{*}\right)_{0}^{++} \oplus F^{+} \oplus F^{-} \\
S^{2} T_{C}^{*}=E^{\prime} \oplus E^{\prime \prime} \oplus\left(S^{2} T^{1,0}\right)^{\perp} \oplus\left(S^{2} T^{0,1}\right)^{\perp} \\
\oplus\{g\}_{c} \oplus\left(S^{2} T^{*}\right)_{0 C}^{++} \oplus F_{C}^{+} \oplus F_{C}^{-}
\end{gathered}
$$

The orthogonal projections $p_{+}$and $p_{-}$of $\left(S^{2} T^{*}\right)^{+-}$onto $F^{+}$and $F^{-}$are equal to $\frac{1}{2}(\mathrm{id}+*)$ and $\frac{1}{2}(\mathrm{id}-*)$, respectively. We remark that, if we change the orientation of the real structures of $X=Q_{4}$, the bundles $F^{+}$and $F^{-}$are simply interchanged.

If $\nu^{\prime}$ is the element of $S_{a}$ considered in $\S 2$, and if we choose the orientation of the real structures of $X=Q_{4}$ for which the elements $\left\{\xi_{2}, \xi_{3}, \xi_{4}, \xi_{5}\right\}$ of $T_{\nu^{\prime}, a}^{+}$defined in $\S 2$ form an oriented orthonormal basis of $T_{\nu^{\prime}, a}^{+}$, then it is easily verified that $F_{C, a}^{+}$is generated by the elements

$$
\begin{gathered}
\left(d w_{1}+i d w_{2}\right) \cdot\left(d \bar{w}_{1}-i d \bar{w}_{2}\right)-\left(d w_{1}-i d w_{2}\right) \cdot\left(d \bar{w}_{1}+i d \bar{w}_{2}\right) \\
+\left(d w_{3}+i d w_{4}\right) \cdot\left(d \bar{w}_{3}-i d \bar{w}_{4}\right)-\left(d w_{3}-i d w_{4}\right) \cdot\left(d \bar{w}_{3}+i d \bar{w}_{4}\right), \\
\left(d w_{1}+i d w_{2}\right) \cdot\left(d \bar{w}_{3}+i d \bar{w}_{4}\right)-\left(d \bar{w}_{1}+i d \bar{w}_{2}\right) \cdot\left(d w_{3}+i d w_{4}\right), \\
\left(d w_{1}-i d w_{2}\right) \cdot\left(d \bar{w}_{3}-i d \bar{w}_{4}\right)-\left(d \bar{w}_{1}-i d \bar{w}_{2}\right) \cdot\left(d w_{3}-i d w_{4}\right)
\end{gathered}
$$

of $\left(S^{2} T^{*}\right)_{C, a}^{+-}$, while $F_{C, a}^{-}$is generated by the elements

$$
\begin{gathered}
\left(d w_{1}+i d w_{2}\right) \cdot\left(d \bar{w}_{1}-i d \bar{w}_{2}\right)-\left(d w_{1}-i d w_{2}\right) \cdot\left(d \bar{w}_{1}+i d \bar{w}_{2}\right) \\
-\left(d w_{3}+i d w_{4}\right) \cdot\left(d \bar{w}_{3}-i d \bar{w}_{4}\right)+\left(d w_{3}-i d w_{4}\right) \cdot\left(d \bar{w}_{3}+i d \bar{w}_{4}\right), \\
\left(d w_{1}+i d w_{2}\right) \cdot\left(d \bar{w}_{3}-i d \bar{w}_{4}\right)-\left(d \bar{w}_{1}+i d \bar{w}_{2}\right) \cdot\left(d w_{3}-i d w_{4}\right), \\
\left(d w_{1}-i d w_{2}\right) \cdot\left(d \bar{w}_{3}+i d \bar{w}_{4}\right)-\left(d \bar{w}_{1}-i d \bar{w}_{2}\right) \cdot\left(d w_{3}+i d w_{4}\right)
\end{gathered}
$$

of $\left(S^{2} T^{*}\right)_{C, a}^{+-}$.

\section{4. $S O(n+2)$-modules and symmetric 2-forms.}

Let $\Gamma$ and $\hat{H}$ be the sets of equivalence classes of irreducible $S O(n+2)$-modules and irreducible $H$-modules (over $C$ ), respectively. If $F$ is a homogeneous unitary Hermitian vector bundle over $X=Q_{n}$, with $n$ $\geq 3$, we denote by $C_{\gamma}^{\infty}(F)$ the isotypic component of the $S O(n+2)$-module $C^{\infty}(F)$ corresponding to $\gamma \in \Gamma$. Let $V_{\gamma}$ be an irreducible $S O(n+2)$-module which is a representative of $\gamma$. Then the $S O(n+2)$-module $C_{\gamma}^{\infty}(F)$ is isomorphic to $k$ copies of $V_{r}$; this integer $k$, the multiplicity of $C_{r}^{\infty}(F)$, denoted by Mult $C_{r}^{\infty}(F)$, is equal to $\operatorname{dim} \operatorname{Hom}_{H}\left(V_{r}, F_{a}\right)$. If $F$ is the trivial 
complex line bundle over $X$, we identify $C^{\infty}(F)$ with $C^{\infty}(X)$ and $C_{r}^{\infty}(F)$ with a submodule $C_{r}^{\infty}(X)$ of $C^{\infty}(X)$. We endow $C^{\infty}(F)$ with the Hermitian scalar product obtained from the scalar product of $F$ and the $S O(n+2)$-invariant volume form $\omega^{n} / n$ ! of $X$. For $\gamma, \gamma^{\prime} \in \Gamma$, with $\gamma \neq \gamma^{\prime}$, the submodules $C_{r}^{\infty}(F)$ and $C_{\gamma^{\prime}}^{\infty}(F)$ of $C^{\infty}(F)$ are orthogonal. If $F_{1}, F_{2}$ are two homogeneous unitary Hermitian vector bundles over $X$, and if $P$ : $\mathscr{F}_{1} \rightarrow \mathscr{F}_{2}$ is a homogeneous differential operator, then we have

$$
P\left(C_{r}^{\infty}\left(F_{1}\right)\right) \subset C_{r}^{\infty}\left(F_{2}\right),
$$

for all $\gamma=\Gamma$ (see $[19, \S 5.3],[5, \S 2])$.

The vector bundle $\otimes^{k} T_{C}^{*}$ is homogeneous and unitary and the Lichnerowicz Laplacian

$$
\Delta=\Delta_{g}: \otimes^{k} \mathscr{T}_{C}^{*} \rightarrow \otimes^{k} \mathscr{T}_{C}^{*}
$$

defined in [12] is a homogeneous differential operator of order 2; for an intrinsic definition of $\Delta$, see $[8, \S 4]$. We recall that the Laplacian $\Delta$ acting on exterior differential forms is equal to the usual de Rham Laplacian of $(X, g)$. The Lichnerowicz Laplacian $\Delta_{g_{0}}$ corresponding to the metric $g_{0}$ $=4 n g$ is related to $\Delta_{g}$ by

(4.1) $\Delta_{g}=4 n \Delta_{g_{0}}$.

We shall need the following two facts proved in $[11, \S 5]$ which hold on any symmetric space of compact type.

Proposition 4.1. (i) If $F$ is a homogeneous sub-bundle of $\otimes^{k} T_{c}^{*}$, then

$$
\nabla \mathscr{F} \subset \mathscr{T}^{*} \otimes \mathscr{F} .
$$

(ii) The Lichnerowicz Laplacian

$$
\Delta_{g_{0}}: C^{\infty}\left(\otimes^{k} T_{C}^{*}\right) \rightarrow C^{\infty}\left(\otimes^{k} T_{C}^{*}\right)
$$

is equal to the Casimir operator of the $S O(n+2)$-module $C^{\infty}\left(\otimes^{k} T_{C}^{*}\right)$.

The vector bundles appearing in the decomposition (3.4), or in the decomposition (3.8) when $n=4$, are all homogeneous sub-bundles of $\otimes^{2} T_{c}^{*}$. Therefore, by Proposition 4.1, (i ), all these bundles are invariant under $\nabla$. Hence, when $n=4$, we have

$$
p_{+} \nabla_{\xi} h=\nabla_{\xi} p_{+} h,
$$


for all $h \in\left(S^{2} \mathscr{T}^{*}\right)^{+-}$and $\xi \in \mathscr{T}$; from the explicit expression for $p_{+}$, it follows that

$$
* \nabla_{\xi} h=\nabla_{\xi} * h,
$$

for all $h \in\left(S^{2} \mathscr{T}^{*}\right)^{+-}$and $\xi \in \mathscr{T}$.

LEMMA 4.1. Assume that $n=4$ and that the real structures of $X$ are oriented. Let $x \in X$ and $\nu \in S_{x}$. Let $\left\{\zeta_{1}, \zeta_{2}, \zeta_{3}, \zeta_{4}\right\}$ be an oriented orthonormal basis of $T_{\nu, x}^{+}$. Then, for $h \in C^{\infty}\left(\left(S^{2} T^{*}\right)^{+-}\right)$we have

$$
(\operatorname{div} * h)\left(J \zeta_{1}\right)=-(d \check{h})\left(\zeta_{2}, \zeta_{3}, \zeta_{4}\right) .
$$

Proof: Since $\left(S^{2} T^{*}\right)^{+-}$is stable under $\nabla$, by (3.2), (4.2) and (3.6) we have

$$
\begin{aligned}
-(\operatorname{div} * h)\left(J \zeta_{1}\right)= & (\nabla * h)\left(\zeta_{2}, \zeta_{2}, J \zeta_{1}\right)+(\nabla * h)\left(\zeta_{3}, \zeta_{3}, J \zeta_{1}\right) \\
& +(\nabla * h)\left(\zeta_{4}, \zeta_{4}, J \zeta_{1}\right) \\
= & \left.* \nabla_{\zeta_{2}} h\right)\left(\zeta_{2}, J \zeta_{1}\right)+\left(* \nabla_{\zeta_{3}} h\right)\left(\zeta_{3}, J \zeta_{1}\right) \\
& +\left(* \nabla_{\zeta_{4}} h\right)\left(\zeta_{4}, J \zeta_{1}\right) \\
= & (\nabla \check{h})\left(\zeta_{2}, \zeta_{3}, \zeta_{4}\right)+(\nabla \breve{h})\left(\zeta_{3}, \zeta_{4}, \zeta_{2}\right)+(\nabla \check{h})\left(\zeta_{4}, \zeta_{2}, \zeta_{3}\right) \\
= & (d \check{h})\left(\zeta_{2}, \zeta_{3}, \zeta_{4}\right) .
\end{aligned}
$$

We now suppose that the integer $n$ is even and we write $n=2 l$. Let $g_{c}$ and $\mathfrak{h}_{c}$ denote the complexifications of the Lie algebras $g$ and $\mathfrak{h}$, respectively. The subgroup $\boldsymbol{T}$ of $S O(n+2)$, which consists of the matrices

$$
\left(\begin{array}{cccc}
R\left(\theta_{0}\right) & 0 & \cdots & 0 \\
0 & R\left(\theta_{1}\right) & \cdots & 0 \\
\vdots & \vdots & \ddots & \vdots \\
0 & 0 & \cdots & R\left(\theta_{l}\right)
\end{array}\right)
$$

with $\theta_{0}, \ldots, \theta_{l} \in \boldsymbol{R}$, is a maximal torus of $S O(n+2)$. The complexification $\mathrm{t}_{C}$ of the Lie algebra $\mathrm{t}$ of $\boldsymbol{T}$ is a Cartan subalgebra of $\mathrm{g}_{\boldsymbol{C}}=\mathfrak{g}_{\mathfrak{D}}(n+2, \boldsymbol{C})$ and of $\mathfrak{h}_{c}$. For $\mu \in \boldsymbol{C}$, we set

$$
L(\mu)=\left(\begin{array}{cc}
0 & -i \mu \\
i \mu & 0
\end{array}\right) .
$$

For $0 \leq j \leq l$, let $\lambda_{j}$ be the linear form on $\mathrm{t}_{c}$ which sends 


$$
\left(\begin{array}{cccc}
L\left(\mu_{0}\right) & 0 & \cdots & 0 \\
0 & L\left(\mu_{1}\right) & \cdots & 0 \\
\vdots & \vdots & \ddots & \vdots \\
0 & 0 & \cdots & L\left(\mu_{l}\right)
\end{array}\right)
$$

with $\mu_{0}, \ldots, \mu_{l} \in \boldsymbol{C}$, into $\mu_{j}$. We write $\alpha_{j}=\lambda_{j}-\lambda_{j+1}$, for $0 \leq j \leq l-1$, and $\alpha_{l}$ $=\lambda_{l-1}+\lambda_{l}$. We choose a Weyl chamber of $\left(g_{c}, t_{c}\right)$ for which the system $\Delta^{+}$of positive roots is equal to

$$
\left\{\lambda_{j}-\lambda_{k} \mid 0 \leq j<k \leq l\right\} \cup\left\{\lambda_{j}+\lambda_{k} \mid 0 \leq j<k \leq l\right\} .
$$

Then $\left\{\alpha_{0}, \alpha_{1}, \ldots, \alpha_{l}\right\}$ is a system of simple roots of $g_{c}$. We choose a Weyl chamber of $\left(\mathfrak{h}_{c}, \mathrm{t}_{C}\right)$ such that $\left\{\alpha_{1}, \ldots, \alpha_{l}\right\}$ is a system of simple roots of $\mathfrak{h}_{c}$. This system of simple roots of $g_{c}$ induces a partial ordering on $t_{c}^{*}$ : elements $\lambda, \lambda^{\prime} \in \mathrm{t}_{c}^{*}$ satisfy $\lambda^{\prime}>\lambda$ if and only if $\lambda^{\prime}-\lambda=\sum_{j=0}^{l} m_{j} \alpha_{j}$, where $m_{0}, \ldots$, $m_{l}$ are integers $\geq 0$, with $m_{0}+\cdots+m_{l}>0$.

The highest weight of an irreducible $S O(n+2)$-module (resp. $H$ module) is a linear form $\Lambda=\sum_{j=0}^{l} h_{j} \lambda_{j}$ on $\mathrm{t}_{c}$, where $h_{0}, h_{1}, \ldots, h_{l}$ are integers satisfying

$$
h_{0} \geq h_{1} \geq \cdots \geq h_{l-1} \geq\left|h_{l}\right| \quad\left(\text { resp. } h_{1} \geq \cdots \geq h_{l-1} \geq\left|h_{l}\right|\right) .
$$

The equivalence class of such an $S O(n+2)$-module (resp. $H$-module) is determined by this weight. We identify $\Gamma$ (resp. $\widehat{H}$ ) with the set of all such linear forms on $t_{c}$.

We suppose throughout the remainder of this section that $n=4$. The fibers at $a$ of the vector bundles appearing in the decomposition (3.8) of $S^{2} T_{C}^{*}$ are irreducible $H$-modules (see [18]). We consider the unit normal $\nu^{\prime} \in S_{a}$ of $\S 2$ and we choose the orientation of the real structures of $X=Q_{4}$ for which the elements $\left\{\xi_{2}, \xi_{3}, \xi_{4}, \xi_{5}\right\}$ of $T_{\nu^{\prime}, a}^{+}$defined in $\S 2$ form an oriented orthonormal basis of $T_{\nu^{\prime}, a}^{+}$. Using the description of these $H$ modules given in $\S 3$, we see that the highest weights of these irreducible $H$-modules are given by the following table:

\begin{tabular}{|c|c||c|c|}
\hline$H$-module & Highest weight & $H$-module & Highest weight \\
\hline \hline$E_{a}^{\prime}$ & $2 \lambda_{0}$ & $E_{a}^{\prime \prime}$ & $-2 \lambda_{0}$ \\
\hline$\left(S^{2} T^{1,0}\right)_{a}^{\perp}$ & $2 \lambda_{0}+2 \lambda_{1}$ & $\left(S^{2} T^{0,1}\right)_{a}^{\perp}$ & $-2 \lambda_{0}+2 \lambda_{1}$ \\
\hline$\{g\}_{C, a}$ & 0 & $\left(S^{2} T^{*}\right)_{0}^{++}, a$ & $2 \lambda_{1}$ \\
\hline$F_{C, a}^{+}$ & $\lambda_{1}+\lambda_{2}$ & $F_{C, a}^{-}$ & $\lambda_{1}-\lambda_{2}$ \\
\hline
\end{tabular}


For $\gamma \in \Gamma$, we denote by $e_{\gamma}$ the eigenvalue of the Casimir operator of the irreducible $S O(6)$-module $V_{r}$. From (4.1) and Proposition 4.1, (ii), we infer that, if $F$ is a homogeneous complex sub-bundle of $\otimes{ }^{k} T_{C}^{*}$ and $\gamma \in \Gamma$, then $C_{r}^{\infty}(F)$ is an eigenspace of $\Delta=\Delta_{g}$ with eigenvalue $16 e_{\gamma}$. Let $\Gamma^{\prime}$ be the set of elements

$$
\begin{aligned}
& \gamma_{r, s}=(2 r+s) \lambda_{0}+s \lambda_{1}, \\
& \gamma_{r, s}^{\prime}=(2 r+s+2) \lambda_{0}+(s+1) \lambda_{1}+\lambda_{2}, \\
& \gamma_{r, s}^{\prime \prime}=(2 r+s+2) \lambda_{0}+(s+1) \lambda_{1}-\lambda_{2}, \\
& \mu_{r, s}^{\prime}=(2 r+s+2) \lambda_{0}+(s+2) \lambda_{1}+2 \lambda_{2}, \\
& \mu_{r, s}^{\prime \prime}=(2 r+s+2) \lambda_{0}+(s+2) \lambda_{1}-2 \lambda_{2}
\end{aligned}
$$

of $\Gamma$, with $r, s \geq 0$. Using Freudenthal's formula, we obtain

(4.3) $\quad e_{\gamma_{0,1}}=1$.

If $\gamma \in \Gamma^{\prime}$ is not equal to 0 or $\gamma_{0,1}$, then we easily verify that

$$
\gamma>\gamma_{0,1},
$$

and hence by Lemma 13.4C of [9], we have

$$
e_{r}>e_{\gamma_{0,1}}=1 \text {. }
$$

From the branching law for $S O(6)$ and $H$ described in Theorem 1.1 of [18], using the table of highest weights of irreducible $H$-modules given above, we obtain (see also $[18, \S 4]$ ):

PROPOSITION 4.2. If $n=4$, for $\gamma \in \Gamma$, the non-zero multiplicities of $C_{r}^{\infty}(F)$, where $F$ is a homogeneous vector bundle over $X$, equal to one of the vector bundles appearing in the decomposition (3.8) of $S^{2} T_{C}^{*}$, are given by Table 1 .

From Proposition 4.2, (4.4) and the previous discussion, we obtain:

PROPOSition 4.3. Let $\gamma$ be an element of $\Gamma$. Then $C_{\gamma}^{\infty}\left(S^{2} T_{C}^{*}\right) \neq 0$ if and only if $\gamma \in \Gamma^{\prime}$. If $\gamma \in \Gamma^{\prime}$ is not equal to 0 or $\gamma_{0,1}$, then $C_{r}^{\infty}\left(S^{2} T_{C}^{*}\right)$ is an eigenspace of $\Delta$ with eigenvalue $>16$. Moreover $C_{\gamma_{0,1}}^{\infty}\left(S^{2} T_{C}^{*}\right)$ is the eigenspace of $\Delta$ with eigenvalue 16.

For $r, s \geq 0$, according to [16], the function $\tilde{f}_{r, s}$ on $X$ defined in $\S 2$ is the highest weight vector of the irreducible $S O(6)$-module $\mathscr{H}_{r, s}=C_{r_{r, s}}^{\infty}(X)$. It follows that $\mathscr{H}_{r, s}$ is the eigenspace of the Laplacian with eigenvalue $16 e_{r r, s}$. Moreover, $C_{r}^{\infty}(X)=0$, when $\gamma \in \Gamma$ is not of the form $\gamma_{r, s}$, for some $r, s \geq 0$. 
Table 1

\begin{tabular}{|c|c|c|}
\hline$F$ & $\gamma \in \Gamma$ & Mult $C_{\gamma}^{\infty}(F)$ \\
\hline $\begin{array}{l}E^{\prime} \\
E^{\prime \prime}\end{array}$ & $\gamma_{r, s} \quad r \geq 1, s \geq 0$ & 1 \\
\hline \multirow[t]{3}{*}{$\begin{array}{l}\left(S^{2} T^{1,0}\right)^{\perp} \\
\left(S^{2} T^{0,1}\right)^{\perp}\end{array}$} & $\gamma_{r, s} \quad r+s \geq 2$ & $\begin{array}{l}2 \text { if } r \geq 2, s=1 \\
\text { or } r=1, s \geq 2 \\
3 \text { if } r, s \geq 2 \\
1 \text { otherwise }\end{array}$ \\
\hline & $\begin{array}{ll}\gamma_{r, s}^{\prime} & \gamma+s \geq 1 \\
\gamma_{r, s}^{\prime \prime} & r+s \geq 1 \\
\end{array}$ & $\begin{array}{l}2 \text { if } r, s \geq 1 \\
1 \text { otherwise }\end{array}$ \\
\hline & $\begin{array}{ll}\mu_{r, s}^{\prime} & r \geq 1, s \geq 0 \\
\mu_{r, s}^{\prime \prime} & r \geq 1, s \geq 0\end{array}$ & 1 \\
\hline$\{g\}_{C}$ & $\gamma_{r, s} \quad r, s \geq 0$ & 1 \\
\hline \multirow[t]{3}{*}{$\left(S^{2} T^{*}\right)_{0}^{++}$} & $\gamma_{r, s} r \geq 1$ or $s \geq 2$ & $\begin{array}{l}2 \text { if } r \geq 1, s=1 \\
3 \text { if } r \geq 1, s \geq 2 \\
1 \text { otherwise }\end{array}$ \\
\hline & $\begin{array}{l}\gamma_{r, s}^{\prime} \quad r, s \geq 0 \\
\gamma_{r, s}^{\prime \prime} \quad r, s \geq 0\end{array}$ & $\begin{array}{l}2 \text { if } r \geq 0, s \geq 1 \\
1 \text { otherwise }\end{array}$ \\
\hline & $\begin{array}{ll}\mu_{r, s}^{\prime} & r \geq 1, s \geq 0 \\
\mu_{r, s}^{\prime \prime} & r \geq 1, s \geq 0 \\
\end{array}$ & 1 \\
\hline $\begin{array}{l}F_{C}^{+} \\
F_{C}^{-}\end{array}$ & $\begin{array}{ll}\gamma_{r, s} & r \geq 0, s \geq 1 \\
\gamma_{r, s}^{\prime} & r, s \geq 0 \\
\gamma_{r, s}^{\prime \prime} & r, s \geq 0\end{array}$ & 1 \\
\hline
\end{tabular}

\section{Totally geodesic surfaces and curvature-like forms.}

Let $x \in X$ and let $\nu$ be a fixed element of $S_{x}$. We write $T_{x}^{+}=T_{\nu, x}^{+}$, $T_{x}^{-}=T_{\nu, x}^{-}$. We henceforth suppose that the dimension $n$ of $X=Q_{n}$ is $z 4$.

We now recall certain results of [2] concerning the totally geodesic surfaces of $X$ passing through $x$.

1) The closed totally geodesic surfaces of $X$ containing $x$, which have constant curvature equal to 4 and which are isometric to $\boldsymbol{C P}^{1}$, are the submanifolds $\operatorname{Exp}_{x} F$, where $F$ is the subspace of $T_{x}$ determined by an orthonormal set $\{\xi, \eta\}$ of elements of $T_{x}^{+}$and spanned by the family

$$
\{\xi+J \eta, J \xi-\eta\} \text {. }
$$


2) The closed totally geodesic surfaces of $X$ containing $x$, which are isometric to a flat torus, are the submanifolds $\operatorname{Exp}_{x} F$, where $F$ is the subspace of $T_{x}$ determined by an orthonormal set $\{\xi, \eta\}$ of elements of $T_{x}^{+}$ and spanned by one of the following families:

(a) $\{\xi, J \eta\}$;

(b) $\left\{\xi-s J \xi, \eta+\frac{1}{s} J \eta\right\}$, where $s \in R^{*}$;

(c) $\{\xi+J \eta, \eta+J \xi\}$.

3) The closed totally geodesic surfaces of $X$ containing $x$, which have constant curvature equal to 1 and which are isometric to the real projective plane $\boldsymbol{R P}^{2}$, are the submanifolds $\operatorname{Exp}_{x} F$, where $F$ is the subspace of $T_{x}$ determined by an orthonormal set $\{\xi, \eta, \zeta, \lambda\}$ of elements of $T_{x}^{+}$and spanned by the family

$$
\{\xi+J \zeta, \eta+J \lambda\} .
$$

Let $N$ be the sub-bundle of $G$ consisting of the elements of $G$ which vanish when restricted to the closed totally geodesic surfaces isometric to one of the following:

(i) $\quad \boldsymbol{C P}^{1}$ with its metric of constant curvature 4 ;

(ii) a flat torus;

(iii) $\boldsymbol{R}^{2}$ with its metric of constant curvature 1.

According to the description of these surfaces given above, an element $\theta$ of $G_{x}$ belongs to $N$ if and only if :

$$
\begin{aligned}
& \theta(\xi+J \eta, J \xi-\eta, \xi+J \eta, J \xi-\eta)=0, \\
& \theta(\xi, J \eta, \xi, J \eta)=0, \\
& \theta\left(\xi-s J \xi, \eta+\frac{1}{s} J \eta, \xi-s J \xi, \eta+\frac{1}{s} J \eta\right)=0, \\
& \theta(\xi+J \eta, \eta+J \xi, \xi+J \eta, \eta+J \xi)=0,
\end{aligned}
$$

for all orthonormal sets $\{\xi, \eta\}$ of elements of $T_{x}^{+}$and all $s \in R^{*}$, and if

$$
\theta(\xi+J \zeta, \eta+J \lambda, \xi+J \zeta, \eta+J \lambda)=0,
$$

for all orthonormal sets $\{\xi, \eta, \zeta, \lambda\}$ of elements of $T_{x}^{+}$. Clearly, $N$ is stable by $J$ and we therefore obtain the decomposition

$$
N=\left(N \cap G^{+}\right) \oplus\left(N \cap G^{-}\right) .
$$

Throughout the remainder of this paper, we consider an element $\theta$ of $N_{x}$ and an arbitrary orthonormal set $\{\xi, \eta, \zeta, \lambda\}$ of elements of $T_{x}^{+}$. 
For all $t \in \boldsymbol{R}$, the vectors $\xi+t \eta$ and $\eta-t \xi$ are orthogonal. From (5.2), it follows that the function

$$
f(t)=\theta(\xi+t \eta, J \eta-t J \xi, \xi+t \eta, J \eta-t J \xi)
$$

vanishes identically. The equality $f^{\prime}(0)=0$ gives us the relation

$$
\theta(\xi, J \xi, \xi, J \eta)=\theta(\eta, J \eta, \xi, J \eta)
$$

The function $u(s)$ of $s \in R^{*}$, whose value at $s$ is equal to the left-hand side of (5.3), vanishes identically. The vanishing of the constant term and of the coefficient of $\frac{1}{s}$ in the expansion of $u(s)$ gives us the equalities

$$
\begin{aligned}
& \theta(\xi, \eta, \xi, \eta)+\theta(J \xi, J \eta, J \xi, J \eta)=2(\theta(\xi, \eta, J \xi, J \eta)+\theta(\xi, J \eta, J \xi, \eta)), \\
& \theta(\xi, \eta, \xi, J \eta)=\theta(\xi, J \eta, J \xi, J \eta)
\end{aligned}
$$

respectively. If $A(\xi, \eta), B(\xi, \eta)$ are the left-hand sides of (5.1) and (5.4) respectively, the equalities

$$
\begin{aligned}
& A(\xi, \eta)+A(\xi,-\eta)+B(\xi, \eta)+B(\xi,-\eta)=0, \\
& A(\xi, \eta)-A(\xi,-\eta)+B(\xi, \eta)-B(\xi,-\eta)=0, \\
& A(\xi, \eta)+A(\xi,-\eta)-B(\xi, \eta)-B(\xi,-\eta)=0
\end{aligned}
$$

imply that

$$
\begin{array}{ll}
(5.10) & \theta(\xi, \eta, \xi, \eta)+\theta(J \xi, J \eta, J \xi, J \eta)+\theta(\xi, J \xi, \xi, J \xi)+\theta(\eta, J \eta, \eta, J \eta)=0 \\
(5.11) & \theta(\xi, \eta, J \eta, \eta)+\theta(\xi, J \xi, J \eta, J \xi)=0 \\
(5.12) & \theta(\xi, \eta, J \xi, J \eta)+\theta(\xi, J \xi, \eta, J \eta)=0
\end{array}
$$

respectively.

LEMMA 5.1. We have

$$
\theta(\xi, J \zeta, \eta, J \zeta)=\theta(J \xi, \zeta, J \eta, \zeta)=0
$$

ProOF: By (5.2), we have the equalities

$$
\theta(\xi+\eta, J \zeta, \xi+\eta, J \zeta)=\theta(\zeta, J \xi+J \eta, \zeta, J \xi+J \eta)=0,
$$

from which we deduce (5.13).

LEMMA 5.2. We have

$$
\begin{array}{ll}
(5.14) & \theta(\xi, \eta, \xi, \eta)+\theta(J \zeta, J \lambda, J \zeta, J \lambda)=0, \\
(5.15) & \theta(\xi, \eta, J \zeta, \eta)+\theta(\xi, J \lambda, J \zeta, J \lambda)=0, \\
(5.16) & \theta(\xi, J \eta, \zeta, J \lambda)=\theta(\xi, \eta, J \zeta, J \lambda)=0 .
\end{array}
$$

PROOF: The left-hand side $A(\xi, \eta)$ of (5.5) vanishes. We write 


$$
\begin{aligned}
& A(\xi, \eta)+A(\xi,-\eta)+A(-\xi, \eta)+A(-\xi,-\eta)=0, \\
& A(\xi, \eta)+A(\xi,-\eta)-A(-\xi, \eta)-A(-\xi,-\eta)=0, \\
& A(\xi, \eta)-A(\xi,-\eta)-A(-\xi, \eta)+A(-\xi,-\eta)=0
\end{aligned}
$$

from these relations, by (5.2), we obtain the equalities (5.14), (5.15) and

$$
\theta(\xi, \eta, J \zeta, J \lambda)+\theta(\xi, J \lambda, J \zeta, \eta)=0
$$

respectively. By Lemma 5.1, we see that

$$
\begin{aligned}
& \theta(\xi+\eta, J \zeta, \xi+\eta, J \lambda)=0, \\
& \theta(\xi, J \zeta, \eta, J \lambda)+\theta(\eta, J \zeta, \xi, J \lambda)=0 .
\end{aligned}
$$

The first Bianchi identity tells us that

$$
\theta(\xi, \eta, J \zeta, J \lambda)+\theta(\eta, J \zeta, \xi, J \lambda)-\theta(\xi, J \zeta, \eta, J \lambda)=0
$$

and so, by (5.17), we have

$$
\theta(\xi, J \lambda, J \zeta, \eta)=\theta(\xi, \eta, J \zeta, J \lambda)=0 .
$$

LEMMA 5.3. If $\theta \in G^{-}$, then we have

$$
\begin{aligned}
& \theta(\xi, \eta, \xi, \eta)=\theta(\zeta, \lambda, \zeta, \lambda) \\
& \theta(\xi, \eta, \xi, \zeta)=-\theta(\lambda, \eta, \lambda, \zeta)
\end{aligned}
$$

PROOF: The relation (5.18) follows from (5.14). Since $\eta+\zeta$ and $\eta-\zeta$ are orthogonal, (5.18) yields the equality

$$
\theta(\xi, \eta+\zeta, \xi, \eta+\zeta)=\theta(\eta-\zeta, \lambda, \eta-\zeta, \lambda)
$$

by (5.18), we now obtain (5.19).

LEMMA 5.4. If $n \geq 5$, we have

$$
\begin{aligned}
& \theta(\xi, \eta, \xi, \zeta)=\theta(\xi, \eta, \zeta, \lambda)=0 \\
& \theta(\xi, \eta, \xi, \eta)=\theta(\xi, \zeta, \xi, \zeta)
\end{aligned}
$$

If moreover $\theta$ belongs to $G^{+}$, then we have

$$
\theta\left(\xi_{1}, \xi_{2}, \xi_{3}, \xi_{4}\right)=0
$$

for all $\xi_{1}, \xi_{2}, \xi_{3}, \xi_{4} \in T_{x}^{+}$.

PROOF By (5.14), if $\mu$ is a unit vector of $T_{x}^{+}$orthogonal to $\xi, \eta, \zeta$ and $\lambda$, we have the equalities

$$
\theta(\xi, \eta+\zeta, \xi, \eta+\zeta)=-2 \theta(J \lambda, J \mu, J \lambda, J \mu)=\theta(\xi, \eta-\zeta, \xi, \eta-\zeta),
$$


which imply that

$$
\theta(\xi, \eta, \xi, \zeta)=0 .
$$

Hence we obtain

$$
\theta(\xi+\lambda, \eta, \xi+\lambda, \zeta)=0,
$$

and so we have

$$
\theta(\xi, \eta, \lambda, \zeta)+\theta(\lambda, \eta, \xi, \zeta)=0
$$

By the first Bianchi identity, we see that

$$
\theta(\xi, \eta, \zeta, \lambda)=0 \text {. }
$$

Since the vectors $\eta+\zeta$ and $\eta-\zeta$ are orthogonal, from (5.20) we infer that

$$
\theta(\xi, \eta+\zeta, \xi, \eta-\zeta)=0
$$

and thus obtain (5.21). From this relation, we deduce the equality

$$
\theta(\xi, \eta, \xi, \eta)=\theta(\zeta, \lambda, \zeta, \lambda)
$$

If $\theta$ belongs to $G^{+}$, by (5.14) we see that

$$
\theta(\xi, \eta, \xi, \eta)=-\theta(\zeta, \lambda, \zeta, \lambda)
$$

and hence that

$$
\theta(\xi, \eta, \xi, \eta)=0 .
$$

This last relation and (5.20) give us (5.22).

LEMMA 5.5. (i) If $n \geq 5$ or if $\theta \in G^{-}$, we have

(5.23) $\theta(\xi, J \xi, \xi, J \xi)=0$,

(5.24) $\theta(\xi, \eta, J \xi, J \eta)=\theta(\xi, J \xi, \eta, J \eta)=\theta(\xi, J \eta, \eta, J \xi)=0$,

(5.25) $\theta(\xi, J \xi, \xi, J \eta)=\theta(\eta, J \eta, \xi, J \eta)=-\theta(\xi, J \xi, \eta, J \xi)$.

(ii) Whenever $\theta \in G^{+}$, we have

(5.26) $\theta(\xi, \eta, \xi, J \eta)=-\theta(\eta, \xi, \eta, J \xi)$,

(5.27) $\theta(\xi, \eta, \xi, J \xi)=\theta(\eta, \xi, \eta, J \eta)$;

if moreover $n \geq 5$, the expressions in the equalities (5.25) all vanish.

(iii) Whenever $\theta \in G^{-}$, we have

(5.28) $\theta(\xi, \eta, \xi, J \eta)=\theta(\eta, \xi, \eta, J \xi)$,

(5.29) $\theta(\xi, \eta, \xi, J \xi)=-\theta(\eta, \xi, \eta, J \eta)$. 
ProOF : Whenever $\theta \in G^{-}$, or whenever $\theta \in G^{+}$and $n \geq 5$, according to the equality (5.22) of Lemma 5.4, the relations (5.8) and (5.10) tell us that

(5.30) $\theta(\xi, \eta, J \xi, J \eta)=\theta(\xi, J \eta, \eta, J \xi)$,

$$
\theta(\xi, J \xi, \xi, J \xi)=-\theta(\eta, J \eta, \eta, J \eta)
$$

respectively. Thus we have

$$
\theta(\xi, J \xi, \xi, J \xi)=-\theta(\zeta, J \zeta, \zeta, J \zeta)=\theta(\eta, J \eta, \eta, J \eta),
$$

and so (5.23) holds. By (5.30), (5.12) and the first Bianchi identity, we obtain (5.24). On the other hand, the relation (5.23) tells us that

$$
f(t)=\theta(\xi+t \eta, J \xi+t J \eta, \xi+t \eta, J \xi+t J \eta)=0,
$$

for all $t \in \boldsymbol{R}$. We write $f^{\prime}(0)=0$ and see that

$$
\theta(\xi, J \xi, \xi, J \eta)+\theta(\xi, J \xi, \eta, J \xi)=0 ;
$$

this equality and (5.7) give us (5.25). If $\theta \in G^{+}$, we have

$$
\theta(\xi, J \xi, \eta, J \xi)=\theta(\xi, J \xi, \xi, J \eta)
$$

and hence all the expressions of (5.25) vanish when $n \geq 5$. The equalities (5.26) and (5.28) are consequences of (5.9); on the other hand, (5.11) implies (5.27) and (5.29).

PROPOSITION 5.1. If $n=4$, we have

(5.31) $\operatorname{Tr}\left(N \cap G^{+}\right) \subset\left(S^{2} T^{*}\right)^{+-}, \quad \operatorname{Tr}\left(N \cap G^{-}\right) \subset E$,

(5.32) $\operatorname{Tr} \cdot \operatorname{Tr} N=\{0\}$.

PROOF : Since $\{J \xi, J \eta, J \zeta, J \lambda\}$ is an orthonormal basis of $T_{x}^{-}$, by (5.2) we have

$$
\begin{aligned}
(\operatorname{Tr} \theta)(\xi, \xi)=\theta(\xi, J \xi, \xi, J \xi)+\theta(\xi, \eta, \xi, \eta) \\
+\theta(\xi, \zeta, \xi, \zeta)+\theta(\xi, \lambda, \xi, \lambda)
\end{aligned}
$$

and so

$$
\begin{aligned}
(\operatorname{Tr} \theta)(\xi, \xi)+(\operatorname{Tr} \theta)(\eta, \eta)=\theta & (\xi, J \xi, \xi, J \xi)+\theta(\eta, J \eta, \eta, J \eta) \\
& +2 \theta(\xi, \eta, \xi, \eta)+\theta(\xi, \zeta, \xi, \zeta) \\
& +\theta(\xi, \lambda, \xi, \lambda)+\theta(\eta, \zeta, \eta, \zeta) \\
& +\theta(\eta, \lambda, \eta, \lambda) .
\end{aligned}
$$

Moreover, by (5.13) we have 


$$
\begin{array}{r}
(\operatorname{Tr} \theta)(\xi, \eta)=\theta(\xi, J \xi, \eta, J \xi)+\theta(\xi, J \eta, \eta, J \eta) \\
+\theta(\xi, \zeta, \eta, \zeta)+\theta(\xi, \lambda, \eta, \lambda)
\end{array}
$$

We now prove the first relation of (5.31). If $\theta \in G^{+}$, according to (5.10), the sum of the first three terms of the right-hand side of (5.34) vanishes. Then by (5.14), we obtain the equality

$$
(\operatorname{Tr} \theta)(\xi, \xi)+(\operatorname{Tr} \theta)(\eta, \eta)=0 .
$$

Therefore we have

$$
(\operatorname{Tr} \theta)(\xi, \xi)=-(\operatorname{Tr} \theta)(\eta, \eta)=(\operatorname{Tr} \theta)(\zeta, \zeta)=-(\operatorname{Tr} \theta)(\xi, \xi),
$$

and so $(\operatorname{Tr} \theta)(\xi, \xi)=0$. Since $\xi$ can be an arbitrary unit vector of $T_{x}^{+}$, by polarization we see that

$$
(\operatorname{Tr} \theta)\left(\eta_{1}, \eta_{2}\right)=0,
$$

for all $\eta_{1}, \eta_{2} \in T_{x}^{+}$. According to (3.2), $\operatorname{Tr} \theta$ belongs to $\left(S^{2} T^{*}\right)^{+-}$. We next verify the second relation of (5.31). If $\theta \in G^{-}$, according to (5.25) the sum of the first two terms of the right-hand side of (5.35) vanishes. Thus by (5.19) we obtain

(5.36) $\quad(\operatorname{Tr} \theta)(\xi, \eta)=0$.

According to (5.33), (5.23) and (5.18), we have

$$
\begin{aligned}
(\operatorname{Tr} \theta)(\xi, \xi) & =\theta(\xi, \eta, \xi, \eta)+\theta(\xi, \zeta, \xi, \zeta)+\theta(\xi, \lambda, \xi, \lambda) \\
& =\theta(\eta, \xi, \eta, \xi)+\theta(\eta, \lambda, \eta, \lambda)+\theta(\eta, \zeta, \eta, \zeta) \\
& =(\operatorname{Tr} \theta)(\eta, \eta) .
\end{aligned}
$$

By Lemma 2.1, from (5.36) and the equality

$$
(\operatorname{Tr} \theta)(\xi, \xi) g(\eta, \eta)-(\operatorname{Tr} \theta)(\eta, \eta) g(\xi, \xi)=0,
$$

we deduce that

$$
\begin{aligned}
& (\operatorname{Tr} \theta)(\xi+s J \xi, \eta+s J \eta)=0, \\
& (\operatorname{Tr} \theta)(\xi+s J \xi, \xi+s J \xi)=(\operatorname{Tr} \theta)(\eta+s J \eta, \eta+s J \eta),
\end{aligned}
$$

for all $s \in \boldsymbol{R}$. Since $\operatorname{Tr} \theta$ belongs to $\left(S^{2} T^{*}\right)^{-}$, we obtain the equalities

$$
(\operatorname{Tr} \theta)(\xi, J \eta)=0, \quad(\operatorname{Tr} \theta)(\xi, J \xi)=(\operatorname{Tr} \theta)(\eta, J \eta) .
$$

We set

$$
a=(\operatorname{Tr} \theta)(\xi, \xi), \quad b=(\operatorname{Tr} \theta)(\xi, J \xi) .
$$

By (5.36), (5.37) and (5.38), it is easily seen that $\operatorname{Tr} \theta$ is equal to the 
element $a h_{\nu}+b h_{J_{\nu}}$ of $E_{x}$. Finally, the equality (5.32) is a direct consequence of (5.31) and the orthogonal decomposition (3.3).

LEMMA 5.6. If $n \geq 5$ and $\theta \in G^{+}$, we have

$$
\theta(\zeta, J \zeta, \xi, J \eta)=\theta(\zeta, \xi, J \eta, J \zeta)=\theta(\zeta, J \eta, \xi, J \zeta)=0 .
$$

ProOF : Since $\theta$ belongs to $G^{+}$, we see that

$$
\begin{aligned}
& \theta(\zeta, J \zeta, \xi, J \eta)=\theta(J \zeta, \zeta, J \xi, \eta)=\theta(\zeta, J \zeta, \eta, J \xi) \\
& \theta(\zeta, \xi, J \eta, J \zeta)=\theta(J \zeta, J \xi, \eta, \zeta)=\theta(\zeta, \eta, J \xi, J \zeta)
\end{aligned}
$$

By (5.24), we have

$$
\theta(\zeta, J \zeta, \xi+\eta, J \xi+J \eta)=\theta(\zeta, \xi+\eta, J \zeta, J \xi+J \eta)=0,
$$

and so, by (5.24) and the equalities (5.40), we see that the two first expressions of (5.39) vanish. The vanishing of the last expression of (5.39) now follows from the first Bianchi identity.

LEMMA 5.7. If $n \geq 5$ and $\theta \in G^{+}$, we have

$$
\begin{aligned}
& \theta(\xi, \eta, \xi, J \xi)=2 \theta(\zeta, \xi, \zeta, J \eta)=2 \theta(\zeta, \xi, \eta, J \zeta) \\
& \theta(\xi, \eta, \zeta, J \zeta)=0 \\
& \theta(\eta, \xi, \eta, J \xi)=\theta(\eta, \zeta, \eta, J \zeta)-\theta(\xi, \zeta, \xi, J \zeta)
\end{aligned}
$$

PROOF : Let $\mu$ be a unit vector of $T_{x}^{+}$orthogonal to $\xi, \eta, \zeta$ and $\lambda$. By (5.15), since $\theta$ belongs to $G^{+}$, we have

$$
\theta(\xi, \eta, J \zeta, \eta)=\theta(J \xi, \lambda, \zeta, \lambda)=\theta(J \zeta, \mu, \xi, \mu)=\theta(J \xi, \eta, \zeta, \eta)
$$

On the other hand, if we consider the orthonormal set $\{\xi,(\eta+\zeta) / \sqrt{2}\}$ of vectors of $T_{x}^{+}$, according to (5.27), we see that

$$
A(\eta)=2 \theta(\xi, \eta+\zeta, \xi, J \xi)-\theta(\eta+\zeta, \xi, \eta+\zeta, J \eta+J \zeta)=0 .
$$

We write $A(\eta)-A(-\eta)=0$ and then, by (5.27), we find that

$$
\theta(\xi, \eta, \xi, J \xi)=\theta(\zeta, \xi, \zeta, J \eta)+\theta(\eta, \xi, \zeta, J \zeta)+\theta(\zeta, \xi, \eta, J \zeta) .
$$

According to (5.26), we know that

$$
\theta(\xi+\eta, \zeta, \xi+\eta, J \zeta)=-\theta(\zeta, \xi+\eta, \zeta, J \xi+J \eta),
$$

and so, by (5.26) and (5.44), we have

$$
\theta(\zeta, \xi, \eta, J \zeta)+\theta(\zeta, \eta, \xi, J \zeta)=2 \theta(\zeta, \xi, \zeta, J \eta) .
$$

The first Bianchi identity tells us that

$$
\theta(\zeta, \xi, \eta, J \zeta)-\theta(\zeta, \eta, \xi, J \zeta)=\theta(\eta, \xi, \zeta, J \zeta),
$$


and hence we have

$$
2 \theta(\zeta, \xi, \eta, J \zeta)=2 \theta(\zeta, \xi, \zeta, J \eta)+\theta(\eta, \xi, \zeta, J \zeta) .
$$

By (5.45) and (5.46), we obtain

$$
2 \theta(\xi, \eta, \xi, J \xi)=4 \theta(\zeta, \xi, \zeta, J \eta)+3 \theta(\eta, \xi, \zeta, J \zeta) .
$$

By (5.27) and (5.44), the expressions $\theta(\xi, \eta, \xi, J \xi)$ and $\theta(\zeta, \xi, \zeta, J \eta)$ are symmetric in $\xi$ and $\eta$. As $\theta(\eta, \xi, \zeta, J \zeta)$ is skew-symmetric in $\xi$ and $\eta$, the relation (5.47) is equivalent to the first equality of (5.41) and (5.42). The second equality of (5.41) now follows from (5.46). Since

$$
\{(\xi+\eta) / \sqrt{2},(\xi-\eta) / \sqrt{2}\}
$$

is an orthonormal set of vectors of $T_{x}^{+}$, according to (5.41), we have

$$
B(\eta)=\theta(\xi+\eta, \xi-\eta, \xi+\eta, J \xi+J \eta)-4 \theta(\zeta, \xi+\eta, \xi-\eta, J \zeta)=0 .
$$

We write $B(\eta)+B(-\eta)=0$, and then, by (5.26), we obtain the relation (5.43).

LEMMA 5.8. If $n \geq 5$ and $\theta \in G^{-}$, we have

$$
\theta(\xi, \eta, \xi, J \eta)=\theta(\xi, \zeta, \xi, J \zeta)
$$

ProOF: By (5.21), we have

$$
\theta(\xi, \eta, \xi, \eta) g(\zeta, \zeta)-\theta(\xi, \zeta, \xi, \zeta) g(\eta, \eta)=0 ;
$$

Lemma 2.1 tells us that the function $f$, whose value at $s \in \boldsymbol{R}$ is

$$
\theta(\xi+s J \xi, \eta+s J \eta, \xi+s J \xi, \eta+s J \eta)-\theta(\xi+s J \xi, \zeta+s J \zeta, \xi+s J \xi, \zeta+s J \zeta),
$$

vanishes identically. The equality $f^{\prime}(0)=0$ and (5.28) give us (5.48).

Let $u$ be an element of $\otimes^{2} T_{x}^{*}$ and $V$ be a subspace of $T_{x}$. Then the following assertions are equivalent : $V$;

(i ) $u\left(\zeta_{1}, \zeta_{1}\right)=u\left(\zeta_{2}, \zeta_{2}\right)$, for any orthonormal set $\left\{\zeta_{1}, \zeta_{2}\right\}$ of vectors of

(ii) $u\left(\zeta_{1}, \zeta_{1}\right)$ is independent of the unit vector $\zeta_{1}$ of $V$.

In fact, if $\left\{\zeta_{1}, \zeta_{2}\right\}$ is an orthonormal set of vectors of $V$, condition (i) tells us that

$$
u\left(\zeta_{1}+\zeta_{2}, \zeta_{1}+\zeta_{2}\right)=u\left(\zeta_{1}-\zeta_{2}, \zeta_{1}-\zeta_{2}\right)
$$

and hence that

$$
u\left(\zeta_{1}, \zeta_{2}\right)+u\left(\zeta_{2}, \zeta_{1}\right)=0
$$


Thus assertion (i ) implies that

$$
u\left(\cos t \cdot \zeta_{1}+\sin t \cdot \zeta_{2}, \cos t \cdot \zeta_{1}+\sin t \cdot \zeta_{2}\right)=u\left(\zeta_{1}, \zeta_{1}\right),
$$

and so (ii) holds. We shall use this criterion in the course of the proof of the following lemma.

Lemma 5.9. Suppose that $n \geq 5$ and that $\theta \in G^{-}$. Then we have

$$
\begin{array}{ll}
(5.49) & \theta(\zeta, \xi, \eta, J \zeta)=-\theta(\zeta, \eta, \xi, J \zeta), \\
(5.50) & \theta(\zeta, J \xi, \eta, J \zeta)=-\theta(\zeta, J \eta, \xi, J \zeta), \\
(5.51) & \theta(\xi, \eta, \zeta, J \zeta)=-2 \theta(\zeta, \xi, \eta, J \zeta), \\
(5.52) & \theta(\zeta, \xi, \zeta, J \eta)=-3 \theta(\zeta, \xi, \eta, J \zeta)-\theta(\xi, \eta, \xi, J \xi), \\
(5.53) & \theta(\zeta, J \zeta, \xi, J \eta)=\theta(\zeta, J \xi, \eta, J \zeta)+\theta(\xi, J \xi, \xi, J \eta), \\
(5.54) & \theta(\zeta, \xi, J \eta, J \zeta)=-2 \theta(\zeta, J \xi, \eta, J \zeta)-\theta(\xi, J \xi, \xi, J \eta),
\end{array}
$$

and the left-hand sides of all these equalities are independent of the choice of the unit vector $\zeta$ of $T_{x}^{+}$orthogonal to $\xi$ and $\eta$.

PRoOF : According to Lemmas 2.1, 5.1 and 5.4, we have

$$
\begin{aligned}
& f_{1}(s)=\theta(J \zeta-s \zeta, \xi+s J \xi, J \zeta-s \zeta, \eta+s J \eta)=0, \\
& f_{2}(s)=\theta(\zeta+s J \zeta, \xi+s J \xi, \zeta+s J \zeta, \eta+s J \eta)=0,
\end{aligned}
$$

for all $s \in \boldsymbol{R}$. We write $f_{1}^{\prime}(0)=0$ and $f_{2}^{\prime}(0)=0$, and then obtain

$$
\begin{gathered}
\theta(J \zeta, J \xi, J \zeta, \eta)+\theta(J \zeta, \xi, J \zeta, J \eta)-\theta(\zeta, \xi, J \zeta, \eta) \\
\quad-\theta(J \zeta, \xi, \zeta, \eta)=0, \\
\theta(\zeta, \xi, \zeta, J \eta)+\theta(\zeta, J \xi, \zeta, \eta)+\theta(\zeta, \xi, J \zeta, \eta)+\theta(J \zeta, \xi, \zeta, \eta)=0,
\end{gathered}
$$

respectively. Since $\theta \in G^{-}$, equation (5.55) gives us

$$
\theta(\zeta, \xi, \zeta, J \eta)+\theta(\zeta, J \xi, \zeta, \eta)-\theta(\zeta, \xi, J \zeta, \eta)-\theta(J \zeta, \xi, \zeta, \eta)=0 .
$$

Taking the difference of equations (5.56) and (5.57), we obtain the relation (5.49). From the first Bianchi identity and (5.49), we deduce (5.51). The equation (5.50) follows directly from the fact that $\theta$ belongs to $G^{-}$. Since $\{\xi,(\eta+\zeta) / \sqrt{2}\}$ is a orthonormal set of elements of $T_{x}^{+}$, by (5.29) we have

$$
A(\eta)=2 \theta(\xi, \eta+\zeta, \xi, J \xi)+\theta(\eta+\zeta, \xi, \eta+\zeta, J \eta+J \zeta)=0 ;
$$

we consider the equality $A(\eta)-A(-\eta)=0$ and by (5.29) find that

$$
\theta(\zeta, \xi, \zeta, J \eta)=\theta(\xi, \eta, \zeta, J \zeta)-\theta(\zeta, \xi, \eta, J \zeta)-\theta(\xi, \eta, \xi, J \xi) .
$$

The relation (5.52) is a direct consequence of the preceding equality and of (5.51). By the first equality of (5.25), we see that 


$$
B(\eta)=2 \theta(\xi, J \xi, \xi, J \eta+J \zeta)-\theta(\eta+\zeta, J \eta+J \zeta, \xi, J \eta+J \zeta)=0 .
$$

We write $B(\eta)-B(-\eta)=0$; by Lemma 5.1 , (5.25) and (5.50), we obtain (5.53). The equation (5.54) now follows from the first Bianchi identity, from (5.53) and (5.50). Since $\theta \in G^{-}$, the equality (5.15) tells us that

$$
\theta(\zeta, \xi, \zeta, J \eta)=-\theta(\lambda, \eta, \lambda, J \xi)
$$

by (5.52), (5.49) and (5.29), we have

$$
\theta(\lambda, \eta, \lambda, J \xi)=-\theta(\lambda, \xi, \lambda, J \eta)
$$

Therefore we obtain

$$
\theta(\zeta, \xi, \zeta, J \eta)=\theta(\lambda, \xi, \lambda, J \eta)
$$

By (5.52) and (5.58), we see that

$$
\theta(\zeta, \xi, \eta, J \zeta)=\theta(\lambda, \xi, \eta, J \lambda)
$$

Thus we have

$$
\theta(\zeta, \xi, \eta, J \zeta) g(\lambda, \lambda)-\theta(\lambda, \xi, \eta, J \lambda) g(\zeta, \zeta)=0,
$$

and so Lemma 2.1 tells us that the function $f$, whose value at $s \in \boldsymbol{R}$ is

$$
\theta(\zeta+s J \zeta, \xi+s J \xi, \eta+s J \eta, J \zeta-s \zeta)-\theta(\lambda+s J \lambda, \xi+s J \xi, \eta+s J \eta, J \lambda-s \lambda),
$$

vanishes identically. According to Lemma 5.1 and (5.20), the equality $f^{\prime}(0)=0$ gives us

$$
\theta(\zeta, J \xi, \eta, J \zeta)+\theta(\zeta, \xi, J \eta, J \zeta)=\theta(\lambda, J \xi, \eta, J \lambda)+\theta(\lambda, \xi, J \eta, J \lambda) ;
$$

thus, by (5.54), we find that

(5.60) $\theta(\zeta, J \xi, \eta, J \zeta)=\theta(\lambda, J \xi, \eta, J \lambda)$.

According to the remark preceding the lemma, the last assertion of the lemma follows from the equalities (5.58), (5.59) and (5.60).

LEMMA 5.10. If $n \geq 5$, we have

(5.61) $\theta(\xi, \eta, \zeta, J \lambda)=0$.

PRoOF : According to (5.41) or to the last assertion of Lemma 5.9, depending on whether $\theta$ belongs to $G^{+}$or to $G^{-}$, and by (5.6), we obtain

$$
\theta(\zeta+\lambda, \xi, \eta, J \zeta+J \lambda)=\theta(\zeta-\lambda, \xi, \eta, J \zeta-J \lambda) ;
$$

hence we have 


$$
\theta(\zeta, \xi, \eta, J \lambda)+\theta(\lambda, \xi, \eta, J \zeta)=0
$$

By (5.20) and Lemma 2.1, we see that

$$
f(s)=\theta(\xi+s J \xi, \eta+s J \eta, \zeta+s J \zeta, \lambda+s J \lambda)=0,
$$

for all $s \in \boldsymbol{R}$. We write $f^{\prime}(0)=0$ and obtain the equality

$$
\theta(J \xi, \eta, \zeta, \lambda)+\theta(\xi, J \eta, \zeta, \lambda)+\theta(\xi, \eta, J \zeta, \lambda)+\theta(\xi, \eta, \zeta, J \lambda)=0 .
$$

The first Bianchi identity tells us that

$$
\theta(\xi, \eta, J \zeta, \lambda)=\theta(J \zeta, \eta, \xi, \lambda)-\theta(J \zeta, \xi, \eta, \lambda)
$$

and thus we have

$$
\begin{aligned}
\theta(J \xi, \eta, \zeta, \lambda)-\theta(J \eta, \xi, \zeta, \lambda)+\theta(J \zeta, \eta, \xi, \lambda) \\
-\theta(J \zeta, \xi, \eta, \lambda)+\theta(\xi, \eta, \zeta, J \lambda)=0 .
\end{aligned}
$$

By (5.62), we see that

$$
\theta(J \xi, \eta, \zeta, \lambda)=-\theta(J \lambda, \eta, \zeta, \xi)=\theta(J \lambda, \eta, \xi, \zeta)=-\theta(J \zeta, \eta, \xi, \lambda) ;
$$

the desired result now follows from these relations and the preceding equality.

Let $\left\{\xi_{1}, \ldots, \xi_{n}\right\}$ be an orthonormal basis of $T_{x}^{+}$. If $n \geq 5$, by (5.2), Lemmas 5.1, 5.2 and 5.4 to 5.10, we see that an element $\theta^{\prime}$ of $N \cap G^{+}$ vanishes if and only if

$$
\theta^{\prime}\left(\xi_{i}, \xi_{n}, \xi_{i}, J \xi_{n}\right)=0
$$

for $i=1, \ldots, n-1$, and

$$
\theta^{\prime}\left(\xi_{i}, \xi_{j}, \xi_{i}, J \xi_{i}\right)=0,
$$

for all $1 \leq i<j \leq n$, and that an element $\theta^{\prime \prime}$ of $N \cap G^{-}$vanishes if and only if, for all $1 \leq i<j \leq n$, we have

$$
\begin{aligned}
& \theta^{\prime \prime}\left(\xi_{i}, J \xi_{i}, \xi_{i}, J \xi_{j}\right)=\theta^{\prime \prime}\left(\xi_{i}, \xi_{j}, \xi_{i}, J \xi_{i}\right)=0, \\
& \theta^{\prime \prime}\left(\xi_{l}, \xi_{j}, \xi_{i}, J \xi_{l}\right)=\theta^{\prime \prime}\left(\xi_{l}, J \xi_{i}, \xi_{j}, J \xi_{l}\right)=0,
\end{aligned}
$$

where $1 \leq l \leq n$ is an arbitrary integer different from $i$ and $j$, and if

$$
\theta^{\prime \prime}\left(\xi_{1}, \xi_{2}, \xi_{1}, \xi_{2}\right)=\theta^{\prime \prime}\left(\xi_{1}, \xi_{2}, \xi_{1}, J \xi_{2}\right)=0 \text {. }
$$

From this result, we deduce the

PROPOSITION 5.2. If $n \geq 5$, we have 


$$
\begin{aligned}
& \operatorname{rank}\left(N \cap G^{+}\right) \leq \frac{1}{2}(n+2)(n-1), \\
& \operatorname{rank}\left(N \cap G^{-}\right) \leq 2 n(n-1)+2 .
\end{aligned}
$$

According to $[6, \S 5]$, the image of $E$ under the morphism of vector bundles

$$
\bar{\tau}: S^{2} T^{*} \rightarrow G
$$

defined by

$$
\begin{aligned}
\hat{\tau}(h)\left(\xi_{1}, \xi_{2}, \xi_{3}, \xi_{4}\right)=\frac{1}{2}\left\{h\left(\xi_{1}, \xi_{3}\right) g\left(\xi_{2}, \xi_{4}\right)+h\left(\xi_{2}, \xi_{4}\right) g\left(\xi_{1}, \xi_{3}\right)\right. \\
\left.-h\left(\xi_{1}, \xi_{4}\right) g\left(\xi_{2}, \xi_{3}\right)-h\left(\xi_{2}, \xi_{3}\right) g\left(\xi_{1}, \xi_{4}\right)\right\},
\end{aligned}
$$

for all $h \in S^{2} T^{*}, \xi_{1}, \xi_{2}, \xi_{3}, \xi_{4} \in T$, is contained in $N \cap G^{-}$. Since

$$
\operatorname{Tr} \hat{\tau}(h)=\frac{1}{2} \operatorname{Tr} h \cdot g+(n-1) h,
$$

for $h \in S^{2} T^{*}$ and $\operatorname{Tr} E=0$, we see that

$$
E \subset \operatorname{Tr}\left(N \cap G^{-}\right) \text {. }
$$

Therefore when $n=4$, by Proposition 5. 1, we obtain the equality

(5.63) $\operatorname{Tr}\left(N \cap G^{-}\right)=E$.

On the other hand, when $n \geq 5$, Proposition 5.2 enables us to determine an explicit complement to the infinitesimal orbit of the curvature in $N$, which contains $\hat{\tau}(E)$ (see [6], Proposition 5.2). Moreover, using this description of $N$, it is easily seen that

$$
\operatorname{Tr}\left(N \cap G^{+}\right)=0
$$

and that (5.63) also holds when $n \geq 5$.

\section{References}

[1] A. BESSE, "Manifolds all of whose geodesics are closed," Ergeb. Math. Grenzgeb., Bd. 93, Springer-Verlag, Berlin, Heidelberg, New York, 1978.

[2] Y. DIENG, Quelques résultats de rigidité infinitésimale pour les quadriques complexes, C. R. Acad. Sci. Paris Sér. I Math. 304 (1987), 393-396.

[3] J. GASQUI, Sur la résolubilité locale des équations d'Einstein, Compositio Math. 47 (1982), 43-69.

[4] J. GASQUI and H. GOLDSCHMIDT, Déformations infinitésimales des espaces riemanniens localement symétriques. I, Adv. in Math. 48 (1983), 205-285.

[5] - Déformations infinitésimales des espaces riemanniens localement symétriques. II. La conjecture infinitésimale de Blaschke pour les espaces projectifs complexes, Ann. Inst. Fourier (Grenoble) 34, 2 (1984), 191-226. 
[6] - Rigidité infinitésimale des espaces projectifs et des quadriques complexes, J. Reine Angew. Math. 396 (1989), 87-121.

[ 7 ] Critère d'exactitude pour les formes de degré 1 sur les quadriques complexes, Bull. Soc. Math. France 117 (1989), 103-119.

[ 8 ] H. GoLDSCHMIDT, The Radon transform for symmetric forms on real projective spaces, Contemp. Math. 113 (1990), 81-96.

[9] J.E. HUMPHREYS, "Introduction to Lie algebras and representation theory," Graduate Texts in Math., Vol. 9, Springer-Verlag, New York, Berlin, Heidelberg, 1972.

[10] S. KoBAYASHI and K. NOMIZU, "Foundations of differential geometry," Vol. II, Interscience Publishers, New York, 1969.

[11] N. KoISO, Rigidity and stability of Einstein metrics - The case of compact symmetric spaces, Osaka J. Math. 17 (1980), 51-73.

[12] A. Lichnerowicz, Propagateurs et commutateurs en relativité générale, Inst. Hautes Etudes Sci. Publ. Math. 10 (1961), 52 pp.

[13] R. Michel, Problèmes d'analyse géométrique liés à la conjecture de Blaschke, Bull. Soc. Math. France 101 (1973), 17-69.

[14] - (a) Un problème d'exactitude concernant bes tenseurs symétriques et les géodésiques, C. R. Acad. Sci. Paris Sér. A 284 (1977), 183-186 ; (b) Tenseurs symétriques et géodésiques, C. R. Acad. Sci. Paris Sér. A 284 (1977), 1065-1068.

[15] B. SMYTH, Differential geometry of complex hypersurfaces, Ann. of Math. 85 (1967), $246-266$.

[16] R. STRICHARTZ, The explicit Fourier decomposition of $L^{2}(S O(n) / S O(n-m))$, Can. J. Math. 27 (1975), 294-310.

[17] C. Tsukamoto, Infinitesimal Blaschke conjectures on projective spaces, Ann. Sci. Ecole Norm. Sup. (4) 14 (1981), 339-356.

[18] - Spectra of Laplace-Beltrami operators on $S O(n+2) / S O(2) \times S O(n)$ and $S p(n+1) / S p(1) \times S p(n)$, Osaka J. Math. 18 (1981), 407-426.

[19] N. WALlACH, "Harmonic analysis on homogeneous spaces," Marcel Dekker, New York, 1973.

Institut Fourier

Université de Grenoble I

B. P. 74

38402 Saint Martin d'Hères

France

Department of Mathematics,

Columbia University

New York, N. Y. 10027

U.S. A. 